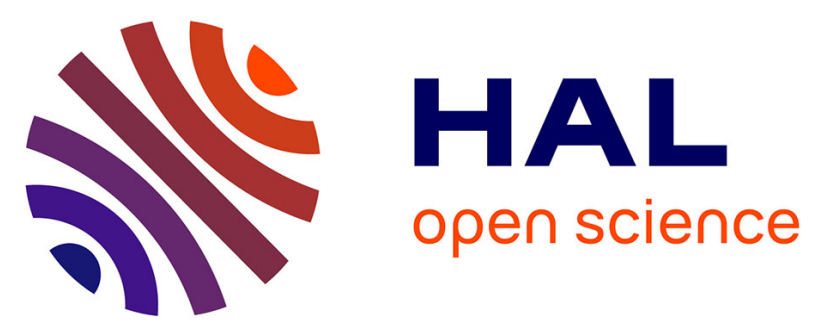

\title{
A new kinetics model to predict the growth of micro-algae subjected to fluctuating availability of light
}

Emna Krichen, Alain Rapaport, Emilie Le Floc'H B, Eric Fouilland

\section{To cite this version:}

Emna Krichen, Alain Rapaport, Emilie Le Floc'H B, Eric Fouilland. A new kinetics model to predict the growth of micro-algae subjected to fluctuating availability of light. Algal Research - Biomass, Biofuels and Bioproducts, 2021, 58, 10.1016/j.algal.2021.102362 . hal-03233538

\section{HAL Id: hal-03233538 \\ https://hal.inrae.fr/hal-03233538}

Submitted on 24 May 2021

HAL is a multi-disciplinary open access archive for the deposit and dissemination of scientific research documents, whether they are published or not. The documents may come from teaching and research institutions in France or abroad, or from public or private research centers.
L'archive ouverte pluridisciplinaire HAL, est destinée au dépôt et à la diffusion de documents scientifiques de niveau recherche, publiés ou non, émanant des établissements d'enseignement et de recherche français ou étrangers, des laboratoires publics ou privés.

\section{(ㅇ)(1) $\$$}

Distributed under a Creative Commons Attribution - NonCommercial - NoDerivatives| 4.0 


\title{
A new kinetics model to predict the growth of micro-algae subjected to fluctuating availability of light
}

\author{
Emna Krichen ${ }^{\mathrm{a}, \mathrm{b}, \mathrm{c}}$, Alain Rapaport ${ }^{\mathrm{a}}$, Emilie Le Floc'h ${ }^{\mathrm{b}}$, Eric Fouilland ${ }^{\mathrm{b}, \mathrm{d}}$ \\ ${ }^{a}$ UMR MISTEA, Univ. Montpellier, INRAE, Institut Agro, Montpellier, France \\ ${ }^{b} U M R$ MARBEC, Univ. Montpellier, CNRS, IFREMER, IRD, Sète, France \\ ${ }^{c} A D E M E$ Agence de l'environnement et de la Maîtrise de l'Energie, Angers, France \\ demail: eric.fouilland@cnrs.fr
}

\begin{abstract}
Light is a key environmental factor for the growth of micro-algae, and optimizing the capture of light is critical for high efficiency production systems. As the density of the population of micro-algae increases, the availability of light decreases, leading to a reduction in the growth rate because of mutual shading, while other effects, such as photo-inhibition, might be especially frequent when the population density is low. Several models in the literature have been developed to take into account light phenomena and predict micro-algal growth, particularly in a mono-culture. With the help of a simple expression for the attenuation of the light, we propose and justify a new growth function that incorporates both photo-inhibition and photolimitation. In agreement with the experimental data, this new formulation describes the micro-algal response to a wide range of situations of excessive or insufficient light intensities through an explicit dependence on both the incident light and the biomass concentration. While simple, the proposed expression can be satisfactorily applied to practical cases under nutrient replete conditions in photo-bioreactors with different sizes and geometries. It extends naturally to the growth of different species, providing a dynamic model which can simulate experiments in a mono-culture as well as in polycultures. The investigation of the competition for light-limited growth shows that the model predicts competitive exclusion, which has also been experimentally demonstrated. This leads to new perspectives for the control and optimization of mixed micro-algal cultures.
\end{abstract}

Keywords: Micro-algae, Modeling, Light availability, Growth rate, Poly-culture, Interactions. 


\section{Introduction}

The study of different aspects related to the behaviour of a micro-algae culture growing in an intensive culture system has gained renewed interest because of the wide fields of application of these photosynthetic microorganisms. Micro-algae are viable sources of biological compounds and constitute a renewable and environmental-friendly feed-stock [1]. Their intensive cultivation is used for the production of high-value bio-products and bio-fuels and also for the treatment of polluted waters. The selection of the appropriate micro-algae species and appropriate methods of culture is essential to guarantee the economic feasibility of the intensive production of micro-algae. Chlorella and Scenedesmus have been considered promising candidates for wastewater treatment $([2,3])$ and bio-fuel production $([2,4,5])$, thanks to their maximum growth rates, biomass yields, and lipid and carbohydrate contents, which can reach high levels.

In a controlled culture system, the growth of micro-algae may be affected by a combination of environmental parameters, such as light intensity, photoperiod, temperature, $\mathrm{pH}$, and composition of the nutrients of the culture system. When nutrients are provided in sufficient quantities and the $\mathrm{pH}$ is maintained at its optimal value, the efficient use of light is essential to optimize and control the growth of an algal culture to ensure the success of industrial production processes, since the light regime and photo-period are critical components that directly affect the production of biomass $([6,7,8,9])$.

Several studies on the effects of light on the growth of micro-algae have been carried out based on experimental as well as theoretical approaches, using fundamental concepts for understanding the dynamic behaviour of lightlimited cultures in photo-bioreactors or outdoor raceways. The proposed mathematical models of micro-algae share, in general, the common objective of having a growth rate as a function of the light irradiance received by micro-algae. According to the typical photosynthesis-irradiance curve ( $\mathrm{P}-$ I curve), describing the response of the rate of photosynthesis to changes in the intensity of the light, three distinct light regimes are depicted. At low intensities, the photosynthesis rate of the algal cells is initially affected by photo-limitation and is usually proportional to the intensity of the light until reaching a saturation point at which the growth rate is at its maximum attainable value and the algae has become light saturated. Beyond 
this point, the growth rate is negatively affected due to photo-inhibition $([10,11,12])$, defined as the degradation of key proteins at high light intensities, which causes a loss of photosynthetic yield and productivity. While photo-inhibition may appear on a short time scale under high irradiance, the response to changes in the long term average irradiance is usually referred to as photo-acclimation $[13,14])$. This phenomenon is linked to the ability of cells to maximize their light absorption capacity under low light and to minimize energy flow under high light by various changes in pigmentation, macro-molecules (e.g. enzymes associated with photosynthesis and respiration), and cell morphology (e.g. cell volume, thylakoids stacking, and transparency) $[15,16,17]$. These two phenomena may affect the $\mathrm{P}-\mathrm{I}$ curve dramatically $[18,19,20]$. The mathematical formulations of the effects of different light phenomena on photosynthesis require more or less complex mechanistic models, depending on the study and the model's application scale.

In most of the studies, incident light is coming from above the algal culture growing in photo-bioreactors. For low-dense cultures it is often assumed that all micro-algae receive the same light intensity. Traditionally, the specific growth rate as a function of the light intensity (P-I curve) is assumed to follow a Monod-like function $[21,22,23]$ or some other non-monotonic expression that accounts for photo-inhibition, such as a Haldane-like function $[24,25,26]$ or the Steele function [27, 28]. However, modeling micro-algae growth in mass culture must consider the response of micro-algae to light intensity and the distribution of the light intensity in the medium. Because the biomass and other light-absorbing substances generate a light gradient in photo-bioreactors, the light intensity that micro-algae can face becomes a function of the depth and biomass concentration within the culture. Thus only micro-algae near the surface receive the light intensity at which the culture is illuminated, while micro-algae located in the darkest zone of the reactor may not receive any light. Light attenuation conditions are affected by biological and physical parameters such as biomass concentration and incident light flux. The light attenuation phenomenon is usually described by the Beer-Lambert law of light absorption [29, 30], according to which the light penetration decreases exponentially with increasing biomass concentrations. Despite the fact that most photo-bioreactor models rely on the BeerLambert law, which is based on the assumption that the light is not scattered in the medium, its use increases the inaccuracy in high-density cultures where multiple scattering events occur $([31,32,33,34])$. The local light availability 
can be calculated using complicated equations accounting for light absorption and scattering in the reactor. However, it is important to note that with more complications (in the expressions of the light distribution or in modeling growth at the cell level), they involve additional input parameters whose determination can be difficult, expensive, or time consuming. Moreover, a large number of parameters can lead to over-fitting, resulting in the model's being poor at predicting the actual trends. In practice, the biomass concentration and the instantaneous light intensity available in the culture medium can be easily monitored, allowing following the light attenuated throughout the cell cultivation period.

When accounting for the impact of light gradients, the average specific micro-algae growth rate can be expressed by summing the local growth rates determined through a biological model, depending on the local light intensities faced by micro-algae cells. This approach can be described using, for example, a Monod-like function coupled with the Beer-Lambert law for the light distribution [32]. Another approach is to describe the average growth rate through a biological model (for instance, the Monod function) that depends on the average light intensity received by the micro-algae (which can be described using the Beer-Lambert law) [28]. This approach assumes that the micro-algae in a well-mixed culture are, on average, exposed to the same light intensity and, therefore, have the same average growth rate [29]. Given that light use by micro-algae is closely related to light attenuation conditions in the bulk of cultivation systems, we evaluate in the present study the accuracy of modeling the algal growth rate as a function of the average attenuated light by cell density. We used two species $C$. sorokiniana and $S$. pectinatus, as candidates for the biological model, growing in one-sided illuminated photo-bioreactors under nutrient replete conditions and constant temperature. The light attenuation inside the culture is assumed to be nonemitting and non-fluorescing, depending on two independent phenomena: (i) absorption by the pigments and (ii) scattering by the whole-cell mass [22]. This light phenomenon was approximated by the summation of the light intensity altered/shaped by the biomass through a simple equation of the form of Michaelis-Menten kinetics (as suggested by [35]), and the incident light intensity (measured perpendicularly to the light source on the boundary of the reactor) modified by the photo-bioreactor and its liquid content. This relationship was validated regardless of the value of the initial light intensity and was an adequate approach, able to cover a wide range of cell concentrations. We then develop a simple growth function explaining the experimental 
results of the response of the process-rate of the micro-algae to a broad range of incident light intensities and biomass concentrations. This new formulation can be considered one of the simplest modeling approaches to describe the behaviour of micro-algal cells in response to light phenomena.

This paper is organized as follows. The influence of the intensity of the incident light and the biomass density on the specific growth rates of the two micr-oalgae candidates (growing in batch cultures) is discussed in Sections 3.1 and 3.2, respectively, through comparisons of the data with classic kinetic models. The light attenuation equation is validated in Section 3.3 and then incorporated in a new growth formulation in Section 3.4, allowing the description of the experimental data sets obtained from the batch cultures. In Section 3.5, the validation of the new kinetic function is presented for the case of continuous light-limited photo-bioreactors using dynamic data for the biomass obtained in mono-cultures and poly-cultures. Finally, in Section 3.6, some cases of the outcome of competition for light are investigated through simulations of the validated multi-species dynamic model under different operating conditions of removal rates and periodic light supply, in continuous mode photo-bioreactors.

\section{Materials and methods}

\section{1. micro-algae strains and pre-culture medium}

The micro-algae were isolated in October 2015 from samples from the high rate algal pond (HRAP) located in the north of France and operated for processing urban wastewater [36, 37]. The isolated species were identified as $C$. sorokiniana and $S$. pectinatus by the Sanger sequencing method [37]. The species were systematically sub-cultured (sub-culturing of $10 \%$ of the inoculum at each cycle) in flasks separately in fresh medium $\mathrm{Z} \mathrm{NH}_{4}(\mathrm{Z} 8$ media [38] buffered with HEPES at $20 \mathrm{mM}$, enriched with ammonium salt $\left(\mathrm{NH}_{4} \mathrm{Cl}\right)$ as the sole nitrogen source, and complemented with sodium carbonate $\left(\mathrm{Na}_{2} \mathrm{CO}_{3}\right)$ to reach a C:N:P ratio of about 88:8:1), and maintained in

laboratory incubators under continuous light $\left(100 \mu \mathrm{E} \mathrm{m}^{-2} \mathrm{~S}^{-1}\right)$ and temperature $25^{\circ} \mathrm{C}$.

\subsection{Experimental procedure and cultivation conditions}

For testing the effects of light on the growth of the biomass for each species, pre-incubations were carried on for 5-day batch cultures under a 
continuous light intensity of $100 \mu \mathrm{E} \mathrm{m}^{-2} \mathrm{~s}^{-1}$ in a $100 \mathrm{~mL}$ flask. Then, each species was diluted (by 2\%,3\%,7\%,10\%,13\%, 20\%, 27\%, 33\%, 40\%, 47\%, $53 \%, 60 \%$ in $40 \mathrm{~mL}$ flasks) with the relevant culture medium where the $\mathrm{pH}$ was maintained constant (at a value of 7.5) in order to test the influence of different biomass concentrations. The incubation of these cultures were carried on for 3-day batch culture in a type 96 microwell plate (Greiner CELLSTARR 96 well plates), filled with the 12 different dilutions with 8 replicates (with a working volume of $250 \mu \mathrm{L}$ per well) for each dilution. Nine identical microwell plates were prepared for each algal species, and then each of them was placed at a fixed position under nine fixed light intensities (from 0 to $900 \mu \mathrm{E} \mathrm{m}^{-2} \mathrm{~S}^{-1}$ ) in four identical laboratory incubators (Panasonic MIR-154-PE) where the temperature was set at $25^{\circ} \mathrm{C}$. The incident light intensities (from cool white Luxeon Rebel LEDs, Lumileds) were measured above and below each microwell plate filled with the culture medium using the scalar PAR sensor ULM 500 Walz.

Thus, a total of 108 combinations of transmitted light intensity and population density were used, including the 12 initial dilutions (equivalent to the diluted initial biomass) and 9 light intensities. The algal growth in the microwell plates was evaluated for each species by fluorescence measurements after $48 \mathrm{~h}$ of exposure to each different condition of both light and biomass concentration outlined above. The specific growth rates $\mu\left(\mathrm{d}^{-1}\right)$ were determined on a total of three biomass measurements (at $t=0 \mathrm{~h}, t=24 \mathrm{~h}$ and $t=48 \mathrm{~h}$ ) using linear least-squares curve fitting on the supplied set of the logarithm of the biomass $\ln (x)$ and time $t$. These growth rates were used for identifying the growth model.

To visualize the changes in the shape of the light attenuation curves according to the cell densities of each species when exposed to several incident light intensities, we selected 9 batch cultures at different stages of growth (non-diluted cultures with different biomass concentrations). Each $40 \mathrm{~mL}$ flask reactor was placed under 8 light levels from cool white LEDs (Luxeon Rebel, Lumileds) delivered from the laboratory incubators (Panasonic MIR154-PE). The light was measured at the centres of the flasks in a water solution with and without cells using the scalar PAR sensor ULM 500 Walz, while the biomass concentrations of each species were determined by optical density $\left(\mathrm{OD}_{650}\right)$ and were then converted to carbon units. Then, for each value of the biomass concentration, the light attenuated by the micro-algal cells can be found as the difference between the two measurements of the light (with and without cells). 
Continuous culturing was carried out in two photo-bioreactors to follow the biomass of the strains over time (in mono-culture or poly-culture) under the same light condition provided by one-sided lighting (using several white fluorescent lamps) at $I_{i n}=165 \mu \mathrm{E} \mathrm{m}^{-2} \mathrm{~s}^{-1}$, and under different initial biomass conditions. These experiments were used to identify the growth model and for the biomass estimation. Each bioreactor consisted of an Erlenmeyer glass vessel of $2 \mathrm{~L}$ with double walls. Between these walls was flowing water thermostatically controlled at $25^{\circ} \mathrm{C}$ (using Thermo Scientifc and VWR circulating bath) allowing maintaining the inoculum temperature constant. The mineral substrate at non/limiting concentrations $(10 \mathrm{~L}$ of sterilized and buffered $\mathrm{Z} 8 \mathrm{NH}_{4}$ culture medium) was introduced continually into the glass vessel at a constant flow by a dual Channel Precision Peristaltic Pump (Ismatec), while the excess of bioreactor liquid was collected in a glass bottle using the same pump, thus keeping the culture volume constant. The reactors were operated at a hydraulic retention time of 4 days (corresponding to a dilution rate of $D=0.25 \mathrm{~d}^{-1}$ ) maintained constant throughout the experiments. To ensure a perfect mixing within the bioreactor, each reactor was agitated at $300 \mathrm{rpm}$ by means of a magnetic system. In addition, a bubbling aeration system was designed as follows: the air is sent into a bottle of water to trap the air particles, an aquarium pump system sends the moisture-saturated air into the culture medium, and then passes through a cannula connected to a transmitting filter of $0.2 \mu \mathrm{m}$ to avoid over-pressure and to limit air contamination. The reactor also has a sampling cannula connected with a non-return valve to minimize the risk of contamination.

\subsection{Analytical procedures}

Batch cultures. In the 3-day batch cultures, monitoring the growth of $C$. sorokiniana and $S$. pectinatus in the microwell plates was carried out daily by fluorescence measurements (EX $450 \mathrm{~nm}, \mathrm{EM} 680 \mathrm{~nm}$ ) and optical density OD at $650 \mathrm{~nm}, 730 \mathrm{~nm}$, and $680 \mathrm{~nm}$ using a micro-plate reader (CHAMELEON, Hidex).

Continuous cultures. In chemostat cultures, samples were collected for cell counts and dissolved nutrient analysis. The cell counts were performed in triplicate using an upright microscope (MOTIC BA310). The algal biomass was also monitored by OD at $650 \mathrm{~nm}$ using a micro-plate reader (FLUOSTAR, BMG Labtech) at $650 \mathrm{~nm}$ through 48 well plates filled daily with 1 $\mathrm{mL}$ of culture sample. 
Carbon conversion. The carbon content was determined as follows: $5-\mathrm{mL}$ samples were filtered onto pre-combusted $\mathrm{AE}$ filters and stored at $80^{\circ} \mathrm{C}$ until the analysis. The filters were dried at $60^{\circ} \mathrm{C}$ for $24 \mathrm{~h}$, pelleted, and analysed using an ANCA mass spectrometer (Europa Scientific).

Referring to batch experiments on the same studied species for different stages of growth with a working volume of $40 \mathrm{~mL}$ under different concentrations of ammonia, a continual light intensity $\left(100 \mu \mathrm{E} \mathrm{m}^{-2} \mathrm{~s}^{-1}\right)$ and a fixed temperature $\left(25^{\circ} \mathrm{C}\right)$ [37], the OD at $650 \mathrm{~nm}$ (measured with CHAMELEON, Hidex) was found to be the best correlated with the Particulate Organic Carbon (POC) content of the cells determined for both species $(\mathrm{POC}=496.14$ $\mathrm{OD}_{650}, R^{2}=0.89$ ).

For the continuous cultures, several samples were collected from both the mono-culture and the poly-culture during chemostat monitoring. The obtained values of the POC allowed establishing a linear correlation between $\mathrm{POC}$ and $\mathrm{OD}_{650}$ (measured with FLUOSTAR, BMG Labtech) $(\mathrm{POC}=208.42$ $\left.\mathrm{OD}_{650}, R^{2}=0.88\right)$.

\subsection{Model identification methods}

First of all, we explored a range of nonlinear models that might be useful for characterizing the growth rate $\mu$ of the studied species according to some classical kinetic functions $(\mu()$.$) from the literature depending on the follow-$ ing variables: the incident light $I_{\text {in }}$ or the biomass $x$. Then we proposed a new kinetic function depending on both these two variables.

The optimal parameters of the growth functions used to explain the characteristics of the growth rates of the algal species (determined in microwell plates) were calibrated using the "fitnlm" function of Matlab, which estimates model parameters and delivers statistics.

To readjust the parameters of the proposed growth function using the data of the biomass of both species in mono-culture (in chemostat), we used the function "fmincon" of Matlab to minimize the least squares criterion:

$\sum_{i=1}^{k} \sum_{j=1}^{n} \frac{\left.\left(\operatorname{Xexp}_{i j}-X s_{i j}\right)_{i j}\right)^{2}}{n}$ where $k=2$ and $n$ is the number of observations of $X_{\text {exp }}$, and $X_{\text {sim }}$ results from the numerical integration of the model (describing the time evolution of the biomass in continuous mode photo-bioreactors) by the "ode45" function of Matlab.

\subsection{Statistical tests}

Statistical results on the identified model parameters were delivered by the "fitnlm" function of Matlab (used to fit nonlinear regression models) and 
are given as param. $\pm \mathrm{SE}$ with an associated $p$-value $p$ for the significance of each regression parameter for $n$ observations. The comparison between the parameters among species for the same growth model was ensured by the same function using the vector of all observations on $\mu$ (for both species) as a response variable, and the matrix of the model variable along with a dummy variable (which takes only the value 0 or 1 according to the species, thus indicating the absence or presence of some categorical effect that may be expected to shift the outcome of the parameter identification) as predictor variables [39]. This involved the need to add to each required parameter a coefficient multiplied by the dummy variable, thus constituting the new model formulation (used in the "fitnlm" function). Then, one can determine the significant differences between the parameters, according to the $p$-value $p$ of these coefficients for $2 n$ observations. The "anova1" function of Matlab (performing one-way analysis of variance) was used once to test the difference between the two species according to their efficiency to attenuate the light in flask reactors experiments (comparison on $K_{h s x}$ parameter among the two species).

\section{Results and discussion}

3.1. Effects of the incident light on the specific growth rate of C. sorokiniana and $S$. pectinatus in batch monoculture

At very low levels of biomass, the average light intensity received by the culture is close to that reaching the reactor surface (i.e. incident light $I_{i n}$ ), particularly for reactors with a small light path. Under these experimental conditions, one can ensure that all cells are exposed to the same light intensity $I_{i n}$. In order to describe accurately the relationship, for each species, of the growth rate $\mu$ with $I_{i n}$, we will use the results obtained experimentally in microwell plates from the lowest concentration of biomass $(1.1 \pm 0.1$ $\left.\mathrm{mgC} \mathrm{L}{ }^{-1}\right)$. We also considered close initial biomass $\left(1.20 \mathrm{mgC} \mathrm{L}^{-1}\right.$ and 1.04 $\mathrm{mgC} \mathrm{L}^{-1}$ for $C$. sorokiniana and $S$. pectinatus, respectively) to compare the growth-light relationships of the two species.

The relationship between $\mu$ and $I_{\text {in }}$ was first compared using a Monod-like kinetics, which assumes that only light limits the growth of the cells. Then we tested the Haldane- and Steele-like models, in which the light inhibition effect at high light intensities is included as well (see Figure 1). The expressions 


\begin{tabular}{|c|c|c|c|c|}
\hline Model $\mu(I)$ & Param. & $C$. & $S$. & $\begin{array}{l}\text { Stat. } \\
\text { comp. }\end{array}$ \\
\hline Monod & $\mu_{m}\left(d^{-1}\right)$ & $1.47^{*} \pm 0.07$ & $1.24^{*} \pm 0.06$ & $* *$ \\
\hline$\frac{\mu_{m} I}{K_{s}+I}$ & $K_{s I}\left(\mu \mathrm{E} \mathrm{m}^{-2} \mathrm{~s}^{-1}\right)$ & $74^{*} \pm 15$ & $95^{*} \pm 18$ & $\mathrm{~ns}$ \\
\hline $\begin{array}{l}\text { Andrews- } \\
\text { Haldane }\end{array}$ & $\mu_{m}\left(d^{-1}\right)$ & $3.15^{*} \pm 0.90$ & $1.56^{*} \pm 0.32$ & $\mathrm{~ns}$ \\
\hline \multirow{2}{*}{$\frac{\mu_{m} I}{K_{s I}+I+\frac{I^{2}}{K_{i I}}}$} & $K_{s I}\left(\mu \mathrm{E} \mathrm{m}^{-2} \mathrm{~s}^{-1}\right)$ & $318^{*} \pm 136$ & $151^{*} \pm 60$ & ns \\
\hline & $K_{i I}\left(\mu \mathrm{E} \mathrm{m}^{-2} \mathrm{~s}^{-1}\right)$ & $726 \pm 382$ & $2834 \pm 2660$ & ns \\
\hline Steele & $\mu_{m}\left(d^{-1}\right)$ & $1.44^{*} \pm 0.03$ & $1.13^{*} \pm 0.03$ & $* *$ \\
\hline$\mu_{m}\left(\frac{I}{I_{m}} e^{\left(1-\frac{I}{I_{m}}\right)}\right)$ & $I_{m}\left(\mu \mathrm{E} \mathrm{m}^{-2} \mathrm{~s}^{-1}\right)$ & $489^{*} \pm 20$ & $489^{*} \pm 27$ & ns \\
\hline
\end{tabular}

Table 1: Summary and comparison of the kinetic parameters, estimated from growth data collected during microplate incubations for C. sorokiniana and S. pectinatus, using Monod, Haldane, and Steele kinetics. The results are represented as param. \pm SE, $n=64$; the statistical comparison was assessed on $2 n$ observations.

* significant regression parameter at $p<0.05(n=128)$,

** significant difference between the parameters of the two species at $p<0.05(n=128)$, ns non-significant difference between the parameters of the two species at $p>0.05(n=$ $128)$.

and parameters of the three kinetic functions obtained from comparison with the data are all summarized in Table 1.

The results show that, over the tested range of incident light intensities, the Monod-like model seems to fit the data of $S$. pectinatus far better than those of $C$. sorokiniana, whose growth appears to be inhibited at high light levels (root mean squared error $\mathrm{RMSE}=0.159$ for $S$. pectinatus $<0.195$ for C. sorokiniana). The determined values of the parameters when using the Monod function to explain the growth rate data of $S$. pectinatus are in line with the results of experiments in previous work performed on the species Scenedesmus caribeanus, which was found to reach a maximum growth rate $\mu_{m}$ of $1.44 \mathrm{~d}^{-1}$ and a half-saturation constant $K_{s I}$ of $68 \mu \mathrm{E} \mathrm{m}^{-2} \mathrm{~s}^{-1}$ [40] ( $\mu_{m}=1.2 \pm 0.1 \mathrm{~d}^{-1}$ and $K_{s I}=95 \pm 18 \mu \mathrm{E} \mathrm{m}^{-2} \mathrm{~s}^{-1}$ in this study).

The reduction in the growth rates of $C$. sorokiniana observed for $I_{\text {in }}>450$ $\mu \mathrm{E} \mathrm{m}^{-2} \mathrm{~s}^{-1}$ suggests its sensitivity to photo-inhibition. This is confirmed by the smaller RMSE obtained when comparing its experimental and simulated data using either the Haldane $(\mathrm{RMSE}=0.173)$ or Steele $(\mathrm{RMSE}=0.183)$ models, both of which have non-monotonic curves which can describe the photo-inhibition phenomenon. The decline in the growth rate of $C$. sorokini- 
ana due to photo-inhibition at high light intensities was also reported in previous studies (at a light intensity of about $250 \mu \mathrm{E} \mathrm{m}^{-2} \mathrm{~s}^{-1}$ and for temperatures $\geq 22^{\circ} \mathrm{C}$ ) [41].

According to the Steele model, both species reach their maximum specific growth rates around an average intensity of $489 \mu \mathrm{E} \mathrm{m}^{-2} \mathrm{~s}^{-1}$, which is supposed to be the optimal light condition under the stated conditions of biomass concentration and temperature.

From Table 1, C. sorokiniana showed the higher maximum specific growth rates compared to those obtained with $S$. pectinatus using either the Monod or Steele kinetics. However, no significant difference was observed between the two species in terms of their affinity to light intensities. This implies that the species' affinities may be similar, or the experimental protocols in this study did not allow determining any difference.

The Haldane-like model provided the lowest RMSE $(\mathrm{RMSE}=0.173$ for C. sorokiniana and RMSE $=0.158$ for $S$. pectinatus) compared with the other two models, thus making it more suitable to represent the data despite the sensitivity of its inhibition constant $K_{i I}$.

According to the model predictions, it appears that $C$. sorokiniana was able to grow more rapidly than $S$. pectinatus when incident light intensities ranged between 100 and $1000 \mu \mathrm{E} \mathrm{m}^{-2} \mathrm{~s}^{-1}$ (see Figure 1). Under the stated experimental conditions, $S$. pectinatus was more resistant than $C$. sorokiniana to photo-inhibition. This is in agreement with previous experiments, showing S. quadricauda with lower photo-inhibition sensitivity than C. sorokiniana under light intensities of about $1000 \mu \mathrm{E} \mathrm{m}^{-2} \mathrm{~s}^{-1}$ [42]. Then, it is expected that the growth rate of $S$. pectinatus will exceed that of $C$. sorokiniana under light intensities higher than $1000 \mu \mathrm{E} \mathrm{m}^{-2} \mathrm{~s}^{-1}$.

From all these observations, it can be seen that the intensity of the incident light can have different effects on the growth of different species of micro-algae. When one species is cultivated under high light intensities and at a low biomass concentration or a reduced light path, photo-inhibition is likely to occur $([43])$. In the case of significant photo-damage, the specific growth rate can be reduced drastically, as shown by several studies $([43,44])$. In poly-culture, the light intensity can favor or disadvantage the growth of one algal species compared to another, depending on its sensitivity to light. Our results suggest that in a mixed culture of the two studied species, $C$. sorokiniana may out-compete $S$. pectinatus under moderate light intensities, but may itself be out-competed by $S$. pectinatus under high light conditions. However, the interactions between these two species may change according to the 
dynamics of their respective biomass during the algal cultivation. Therefore, the interaction between the incident light and the population density was further investigated.
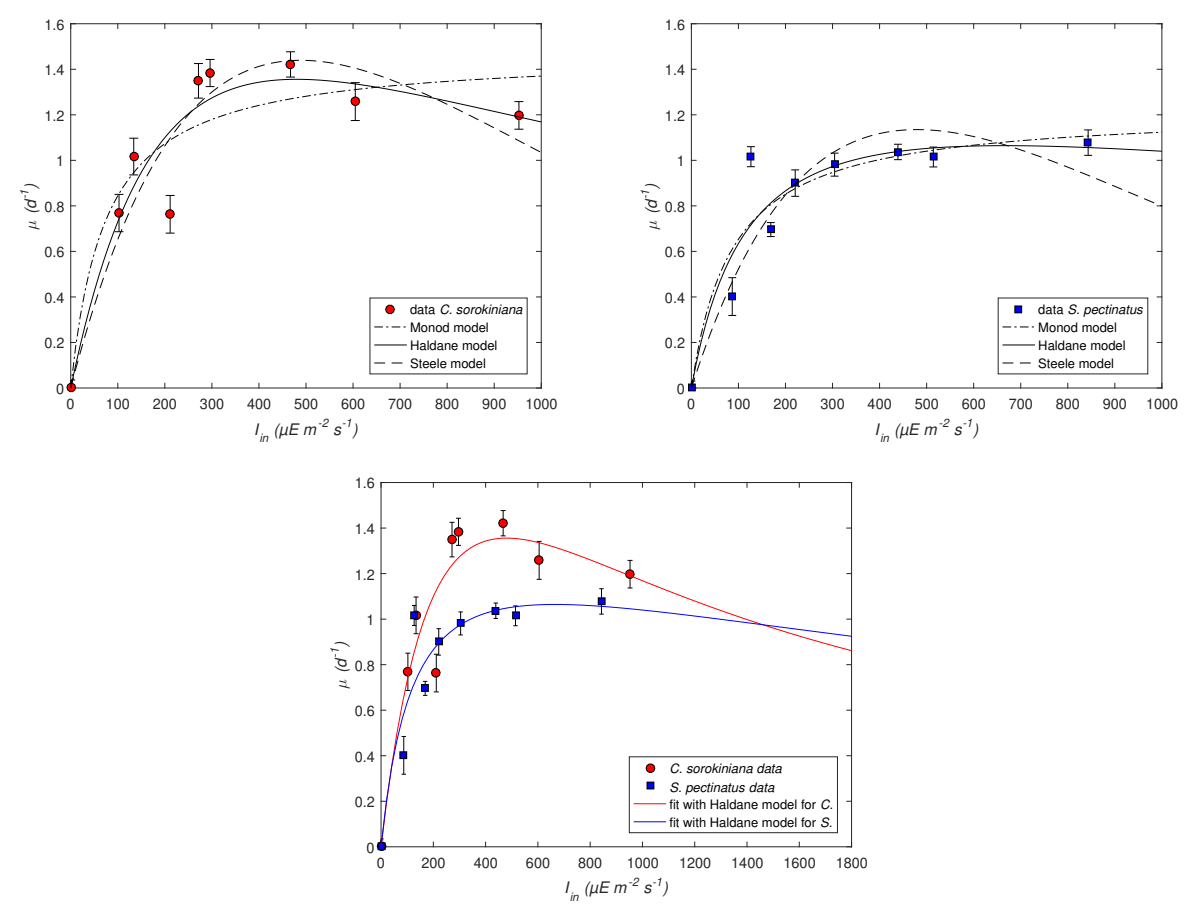

Figure 1: The growth-light relationship for C. sorokiniana and S. pectinatus obtained by the comparison of the growth data, collected from microplate incubations, with the kinetic functions of Monod, Haldane, and Steele. The data of growth rates are reported as means with standard deviations of 8 replicate measurements obtained at an initial biomass concentrations around $1.1 \pm 0.1 \mathrm{mgC} \mathrm{L}^{-1}$ for both species.

3.2. Effects of the density of the biomass on the growth rates of C. sorokiniana and $S$. pectinatus in batch mono-culture

The influence of different biomass levels on the growth of $C$. sorokiniana and $S$. pectinatus was studied. A set of batch tests was performed in microwell plates exposed to 12 initial biomass concentrations between 0.5 and $35 \mathrm{mgC} \mathrm{L}{ }^{-1}$. We here show the data obtained under a fixed incident light $\left(467 \mu \mathrm{E} \mathrm{m}^{-2} \mathrm{~s}^{-1}\right.$ and $439 \mu \mathrm{E} \mathrm{m}^{-2} \mathrm{~s}^{-1}$ for the cultures of $C$. sorokiniana and 


\begin{tabular}{lllll}
\hline Model $\mu(x)$ & Param. & $\boldsymbol{C .}$ & $\boldsymbol{S .}$ & $\begin{array}{l}\text { Stat. } \\
\text { comp. }\end{array}$ \\
\hline $\begin{array}{l}\text { Exponential } \\
\text { declining shape }\end{array}$ & $a$ & $1.52^{*} \pm 0.03$ & $1.02^{*} \pm 0.009$ & ${ }^{* *}$ \\
$a e^{(-b x)}$ & $b$ & $0.06^{*} \pm 0.002$ & $0.036^{*} \pm 0.001$ & ${ }^{* *}$ \\
\hline $\begin{array}{l}\text { Contois }[45] \\
\frac{A}{1+B x}\end{array}$ & $A=\mu_{m}$ & $1.75^{*} \pm 0.03$ & $1.1^{*} \pm 0.01$ & ${ }^{* *}$ \\
\hline
\end{tabular}

Table 2: Summary and comparison of the kinetic parameters, estimated from growth data collected during microplate incubations for $C$. sorokiniana and $S$. pectinatus, depending on biomass density. The results are represented as param. $\pm \mathrm{SE}, n=96$; the statistical comparison was assessed on $2 n$ observations.

* significant regression parameter at $p<0.05$,

** significant difference between the parameters of the two species at $p<0.05$.

S. pectinatus, respectively) for which both species showed maximal growth rates, as described in Section 3.1.

Two classic models were adjusted to the experimental data: a generic model of an exponential declining shape and a model inspired by the densitydependent growth kinetic of Contois, both depending on the biomass density, affecting negatively species specific growth rates. The models' expressions and parameters are summarized in Table 2.

Figure 2 shows the kinetic data of $C$. sorokiniana against those of $S$. pectinatus as functions of the initial biomass concentrations. The growth rates of the two cultures decreased sharply with increasing biomass levels in microplates. The maximal growth rate was obtained for low biomass concentrations. Under this condition, cell sedimentation and subsequent areas of strong light absorption were likely to be reduced, leading to optimal light absorption in the culture system. The decrease of growth rates with increasing biomass densities is likely to be related to light attenuation and cell distribution in the culture volume. Without excluding it, a growth limitation by $\mathrm{CO}_{2}$ is unlikely because the culture medium was supplemented with high $\mathrm{HCO}_{3}^{-}$and the growth rates were determined within the first $48 \mathrm{~h}$ of incubation, thus excluding any substrate limitation. A similar trend in declining growth in dense algal culture has been reported for Scenedesmus sp. and Chlorella sp. due to attenuation of the light [40]. Moreover, previous studies reported that the growth of micro-algae Chlorella sp. was low under insufficient or excessive light intensities ([46], [47]), which is also confirmed by 
our results. Table 2 shows that there is a significant difference between the species' specific growth rates, as stated in Section 3.1.

The change in the species' growth performances with the culture density suggests that at non-inhibiting incident light intensities, C. sorokiniana growth is more efficient than $S$. pectinatus at low biomass levels $\left(<5 \mathrm{mg} . \mathrm{L}^{-1}\right)$. At intermediate levels of biomass (between 5 and $30 \mathrm{mg} . \mathrm{L}^{-1}$ ), the growth of both species was similar. However, under higher biomass densities, S. pectinatus grew more rapidly than $C$. sorokiniana (as shown in Figure 2).

These observations suggest that in the case of poly-culture, S. pectinatus may perform well at high biomass densities despite the relatively low growth rates usually observed, because this species can out-compete light-limited species under low light. However, C. sorokiniana may perform better under clear waters and compete more effectively at moderate light conditions but may lose its advantage as the culture density increases over time. Consequently, the biomass level within a culture is a key factor that can explain the predominance of one species over another when growing together under non-inhibiting light conditions.
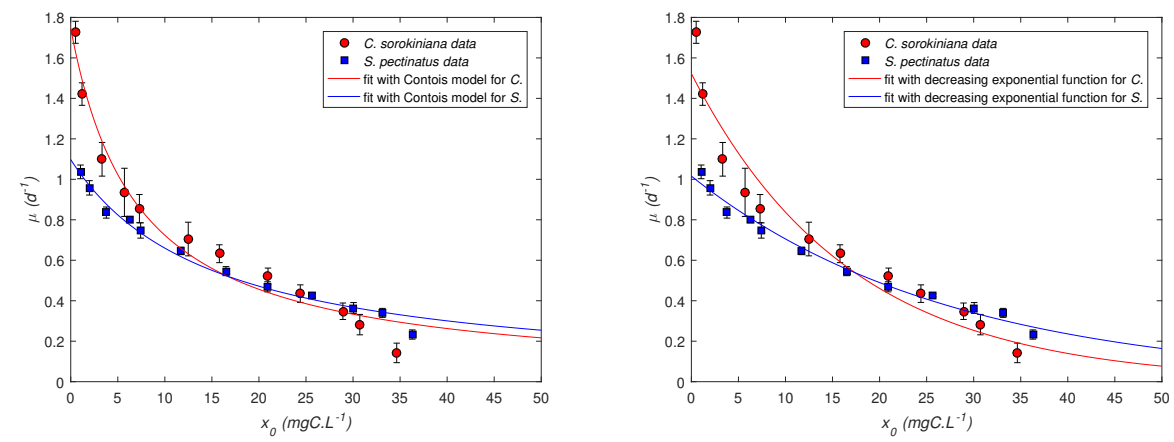

Figure 2: The growth-biomass density relationship for C. sorokiniana and S. pectinatus obtained from microplate incubations. The data of growth rates are reported as means with standard deviations of 8 replicate measurements obtained under a continuous incident light intensity of $467 \mu \mathrm{E} \mathrm{m}^{-2} \mathrm{~s}^{-1}$ for C. sorokiniana and $439 \mu \mathrm{E} \mathrm{m}^{-2} \mathrm{~s}^{-1}$ for $S$. pectinatus.

\subsection{Modeling the light attenuation within cultures}

Light attenuation had significant effects on micro-algae growth. For a one-sided illuminated photo-bioreactor with a fixed light intensity $I_{i n}$, the photo-synthetically active light is a maximum near the liquid boundary in front of the light supply and decreases on passing through the water column. 
In addition to the effect of the depth, and the reflection and refraction at the interfaces boundaries, the absorption of the biomass when it is at high concentrations can induce light limitation within a well-mixed photo-bioreactor. Because of the high pigment content of micro-algae, light is stronly absorbed as it penetrates the culture depth. Under well-mixed conditions, we assumed that i) the individual cells are not stationed exclusively in the light or dark zones of the culture but exposed, on average, to the same light condition in the culture with respect to the instantaneous cell concentration $x$ ii) the average micro-algal growth rate is closely related to light attenuation $I_{a t t x}$ conditions in a given bulk of cultivation system. iii) $I_{\text {attx }}$ is defined as the light dissipated by the summation of both absorption and scattering and given as a function of the biomass concentration

$$
I_{a t t x}\left(I_{i n}, x\right)=\alpha I_{i n} \frac{x}{x+K_{h s x}}
$$

where $K_{h s x}$ (biomass unit) is the half-saturation constant of the biomass concentration $x$ (biomass unit) and $\alpha(\%)$ is the percentage of the maximum effective light available for the growth of the micro-algae.

This model was validated in well-mixed batch reactors (flasks of $40 \mathrm{~mL}$ ) illuminated at several initial light intensities $I_{\text {in }}$ for both studied strains using cultures at different stages of growth (corresponding to different biomass concentration obtained at different incubation times $t_{f}$ ). The light irradiance profiles were determined by plotting the light attenuation $\left(I_{t_{0}}-I_{t_{f}}\right)$ measured at the centres of the flask reactors $\left(\mathrm{I}_{t_{0}}\right.$ without biomass and $I_{t_{f}}$ with biomass at tf) against the biomass concentrations (measured by $\mathrm{OD}_{650}$ and then converted to $\mathrm{mgC} \mathrm{L}^{-1}$ ).

As shown in Figure 3, the higher is $I_{i n}$, the greater is $I_{a t t x}$. The light curve tends towards the irradiance value $\alpha I_{\text {in }}$ measured at the centre of the reactor when filled with only the culture medium. The shape of the obtained graphs appears to be similar to that of the Monod function and was then used to describe the light attenuation phenomenon.

We defined the total light attenuation $I_{a t t}$ within a photo-bioreactor as the summation of the light attenuation by biomass $I_{\text {attx }}$ (including both absorption and scattering) and the light modified by the reactor and its liquid content $I_{a t t 0}=I_{i n}(1-\alpha)$, as summarized in the following expression:

$$
I_{a t t}\left(I_{i n}, x\right)=I_{a t t 0}+I_{a t t x}=I_{i n}\left(1-\alpha\left(1-\frac{x}{x+K_{h s x}}\right)\right)
$$



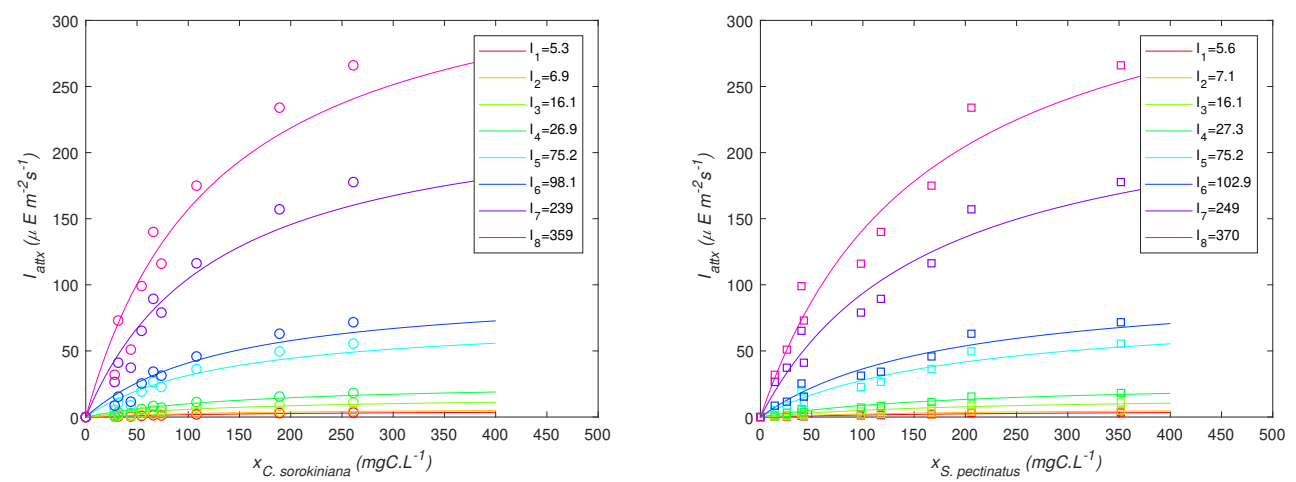

Figure 3: Simulation of the attenuated light model $I_{\text {attx }}$ compared to data obtained in monocultures of $C$. sorokiniana and $S$. pectinatus growing in flasks at different stages of growth and biomass concentrations.

The parameter $\alpha$ can be interpreted as a characteristic of the photobioreactor. This parameter may be estimated with an experimental test carried out with the culture device filled with the culture medium before inoculation. Consequently, the contribution of the reactor and its liquid content to the attenuation of $I_{\text {in }}$ can be given by the absorbed light $I_{\text {in }}-I_{\text {out }}$ (both measured perpendicularly to the light source on either side of the reactor) divided by $I_{i n}$. Then, $\beta=1-\alpha$ represents the percentage of the light unavailable for algal growth, and depends on the wall and depth of the reactor, the transparency of the culture medium, and also the geometry and material of the reactor (such as the reflection and refraction of the light through the walls and at the interface with the medium, which may differ).

For all tested values of $I_{i n}$, the model (1) fits well the measured data for both strains (Figure 3) with different values of $K_{h s x}$ (ANOVA test $p<0.05$; $K_{h s x}=155 \pm 25 \mathrm{mgC} \mathrm{L}^{-1}$ for C. sorokiniania and $K_{h s x}=201 \pm 33 \mathrm{mgC} \mathrm{L}^{-1}$ for S. pectinatus; (param. $\left.\pm \mathrm{SE}, \mathrm{n}:\left[\begin{array}{l}8 \\ 8\end{array}\right]\right)$ ). This suggests that C. sorokiniania can attenuate light more effectively than $S$. pectinatus.

\subsection{Coupling the photo-inhibition and photo-limitation effects in micro-algal growth kinetics}

Based on the previous results, we suppose that the micro-algae growth is affected by both photo-inhibition and photo-limitation, suggesting that a good kinetic model would depend on $I_{\text {in }}$ and $x$. Thus we looked for one model which can represent all the experimental data, by trying to find a function 
that could relate $\mu$ to $I_{\text {att }}$. Although the curve shapes of the growth rates plotted against $I_{a t t}$ resemble the classical Monod-, Haldane-, or Steele-type functions (see the experimental data for $\mu$ in Figures 4 and 5 for $C$. sorokiniana and $S$. pectinatus, respectively), there were no unique sets of parameters that could explain all the experimental data sets. However, one can compute the correlations between the individual parameters identified from one experiment to another. The most remarkable correlation observed for any tested type of kinetics was between $\mu_{m}$ and the tested $x$ condition, when taking $\mu_{m}$ as a decreasing function of $x$. Thus, we propose the following expression:

$$
\mu_{m}(x)=\bar{\mu} \alpha\left(1-\frac{x}{x+K_{h s x}}\right)=\bar{\mu}\left(\alpha-\frac{I_{a t t x}}{I_{\text {in }}}\right)
$$

where $\bar{\mu}$ is the maximal value of the species' specific growth rate.

We built the following kinetic model using (2) and (3)

$$
\mu\left(I_{i n}, x\right)=\mu_{m}(x) \frac{I_{a t t}\left(I_{i n}, x\right)}{K_{s I_{a t t}}+I_{a t t}\left(I_{i n}, x\right)}\left(1-\frac{I_{a t t}\left(I_{i n}, x\right)}{I_{0}}\right)
$$

with $K_{s I_{a t t}}$ the half-saturation constant of attenuated light $\left(\mu \mathrm{E} \mathrm{m}^{-2} \mathrm{~s}^{-1}\right)$ and $I_{0}$ the light intensity $\left(\mu \mathrm{E} \mathrm{m}^{-2} \mathrm{~s}^{-1}\right)$ for which $\mu$ takes the value of 0 for any large enough value of $x$.

With this model we implicitly consider that the inequality

$$
I_{0}>(1-\alpha) I_{\text {in }}
$$

is fulfilled (otherwise no growth is possible). Then, we consider the number

$$
\bar{x}:= \begin{cases}K_{h s x} \frac{I_{0}-(1-\alpha) I_{i n}}{I_{i n}-I_{0}} & \text { if }(1-\alpha) I_{\text {in }}<I_{0}<I_{\text {in }} \\ +\infty & \text { if } I_{0} \geq I_{\text {in }}\end{cases}
$$

which defines a threshold on the biomass level under which growth is possible. Finally, the model writes

$$
\mu\left(I_{i n}, x\right)= \begin{cases}\mu_{m}(x) \frac{I_{a t t}\left(I_{i n}, x\right)}{K_{s I_{a t t}}+I_{a t t}\left(I_{\text {in }}, x\right)}\left(1-\frac{I_{a t t}\left(I_{i n}, x\right)}{I_{0}}\right), & x<\bar{x} \\ 0, & x \geq \bar{x}\end{cases}
$$

under Hypothesis (5).

The Appendix gives the mathematical analysis of the monotonicity of expression (4) with respect to $x$. We show that, over the interval $(0 ; \bar{x})$, 
the function $\mu$ is either decreasing, or increasing and then decreasing with respect to $x$ (see $\mu$ as a function of $x$ in Figures 4 and 5 where $I_{i n}$ is fixed with $\left.I_{\text {in }} \leq I_{0}\right)$.

As shown in Figures 4 and 5, the highest growth rates are obtained with the lowest biomass concentration related to lower light attenuation conditions, which induces a higher light amount received per cell. The effect of light on growth rates was less pronounced at high biomass concentrations than at low concentrations. Interestingly, the greater is the initial biomass concentrations, the higher is the light photo-inhibition despite the high light attenuation by the cells. This suggests that at initial high biomass concentrations, algal cells are responding to high incident light as low light-adapted cells [48]. Therefore, the proposed growth function can indirectly describe algal-cells adaptation to rapidly changing lighting conditions. The model (6) allows describing both the light inhibition effect and the light attenuation effect, and applies to a broad range of incident light intensities $(0-1000 \mu \mathrm{E}$ $\left.\mathrm{m}^{-2} \mathrm{~s}^{-1}\right)$ and biomass densities $\left(0-35 \mathrm{mgC} \mathrm{L}^{-1}\right)$. The model parameters were identified for each species and are presented in Table 3.

\begin{tabular}{llll}
\hline Param. & $\boldsymbol{C}$. & $\boldsymbol{S}$. & Stat. comp. \\
\hline$\alpha$ & $0.75^{*} \pm 0.03$ & $0.81^{*} \pm 0.03$ & ns \\
$\bar{\mu}\left(\mathrm{d}^{-1}\right)$ & $3.25^{*} \pm 0.20$ & $1.75^{*} \pm 0.08$ & $* *$ \\
$K_{\text {sI }}\left(\mu \mathrm{E} \mathrm{m}^{-2} \mathrm{~s}^{-1}\right)$ & $52^{*} \pm 6$ & $26^{*} \pm 3$ & $* *$ \\
$K_{h s x}\left(\mathrm{mgC} \mathrm{L}^{-1}\right)$ & $9.89^{*} \pm 0.31$ & $17.07^{*} \pm 0.53$ & $* *$ \\
$I_{0}\left(\mu \mathrm{E} \mathrm{m}^{-2} \mathrm{~s}^{-1}\right)$ & $1068^{*} \pm 41$ & $1836^{*} \pm 168$ & $* *$ \\
\hline
\end{tabular}

Table 3: Summary and comparison of the new model parameters used in the modeling of C. sorokiniana and $S$. pectinatus growth depending on both incident light intensity and biomass density (in microwell plates). The results are represented as param. $\pm \mathrm{SE}, n=864$; the statistical comparison was assessed on $2 n$ observations.

* significant regression parameter at $p<0.05$,

** significant difference between the parameters of the two species at $p<0.05$, ns non-significant difference between the parameters of the two species at $p>0.05$.

All the estimated parameters show that there are significant differences between the species, except for $\alpha$. We recall that $\alpha$ is a characteristic parameter of the reactor that reflects the contribution of the culture device in the attenuation of $I_{i n}$. Then, it is suggested that this parameter is probably the same in the microwell plates and the maximum effective light available for micro-algae growth always equals $\alpha I_{i n}$. For the maximal value of the species' specific growth rate, the greater $\bar{\mu}$, estimated for $C$. sorokiniana, shows its 
ability to grow faster than $S$. pectinatus when growing conditions are favorable, as suggested in Sections 3.1 and 3.2 in microwell plates experiments. Moreover, the greater $I_{0}$, found for $S$. pectinatus, demonstrates its strongest resistance to high light intensities, which supports our previous results in Section 3.1. We note that the half-saturation constants $K_{s I_{a t t}}$ for the two species were also different. Similarly, the significant difference of $K_{h s x}$ between the two species reflects different responses to the attenuation effect, as stated above (see Section 3.3). However, we notice that the value of $K_{h s x}$ identified for microwell plate cultures was not of the same order of magnitude as that for flask cultures (cf. Section 3.3). This may be explained by the spatial heterogeneity effect related to mixing. In fact, the cells initially suspended in the small volume of few micro-litres $(250 \mu L)$ in the microwell plates tend to accumulate at the bottom of the well, which is not the case for the instantaneous measurement of the light in a perfectly mixed flasks (40 $\mathrm{mL}$ ). This may result in a significant density inhibitory effect on $\mu_{m}$ (following equation (3)) caused by the high spatial heterogeneity, thereby explaining the small value obtained for $K_{h s x}$ in micro-plates. Then, $K_{h s x}$ will increase with the degree of mixing when the same culture device is considered. In addition, we observed higher values of $K_{h s x}$ for $S$. pectinatus compared to C. sorokiniana, whether in microplate or flasks. This is probably due to the differences in shapes and sizes of the cells between the two species. Having the same biomass concentration, a small number of voluminous cells (such as $S$. pectinatus) would attenuate less light than small cells at a much larger number (as is the case for $C$. sorokiniana). Therefore, $K_{h s x}$ would be related to both the species' bio-volumes and the mixing.

We note that different trends are found when comparing the microalgal growth rates using the relevant values of $K_{h s x}$ previously identified for each culturing device (microplates and flasks). The model predicts a drastic reduction of microalgal growth in microwell plates leading to a division of the highest growth rate by a factor of 5 for $C$. sorokiniana and 3 for S. pectinatus, at a density of $35 \mathrm{mgC} \mathrm{L}{ }^{-1}$ and $I_{i n}=450 \mu \mathrm{E} \mathrm{m}^{-2} \mathrm{~s}^{-1}$ (cf. Figures 2, 4, and 5). However, the decline becomes insignificant (division factor of only 1.25 and 1.1 for $C$. sorokiniana and S. pectinatus, respectively) in flasks under the same conditions (taking the same growth parameters of Table 3 except for $\left.K_{h s x}\right)$. These outcomes support that the lack of mixing and the subsequent heterogeneous cell settlement at the bottom of microwell plates may explain such discrepancies.

The new kinetic function (6) highlights the interactions between the inci- 

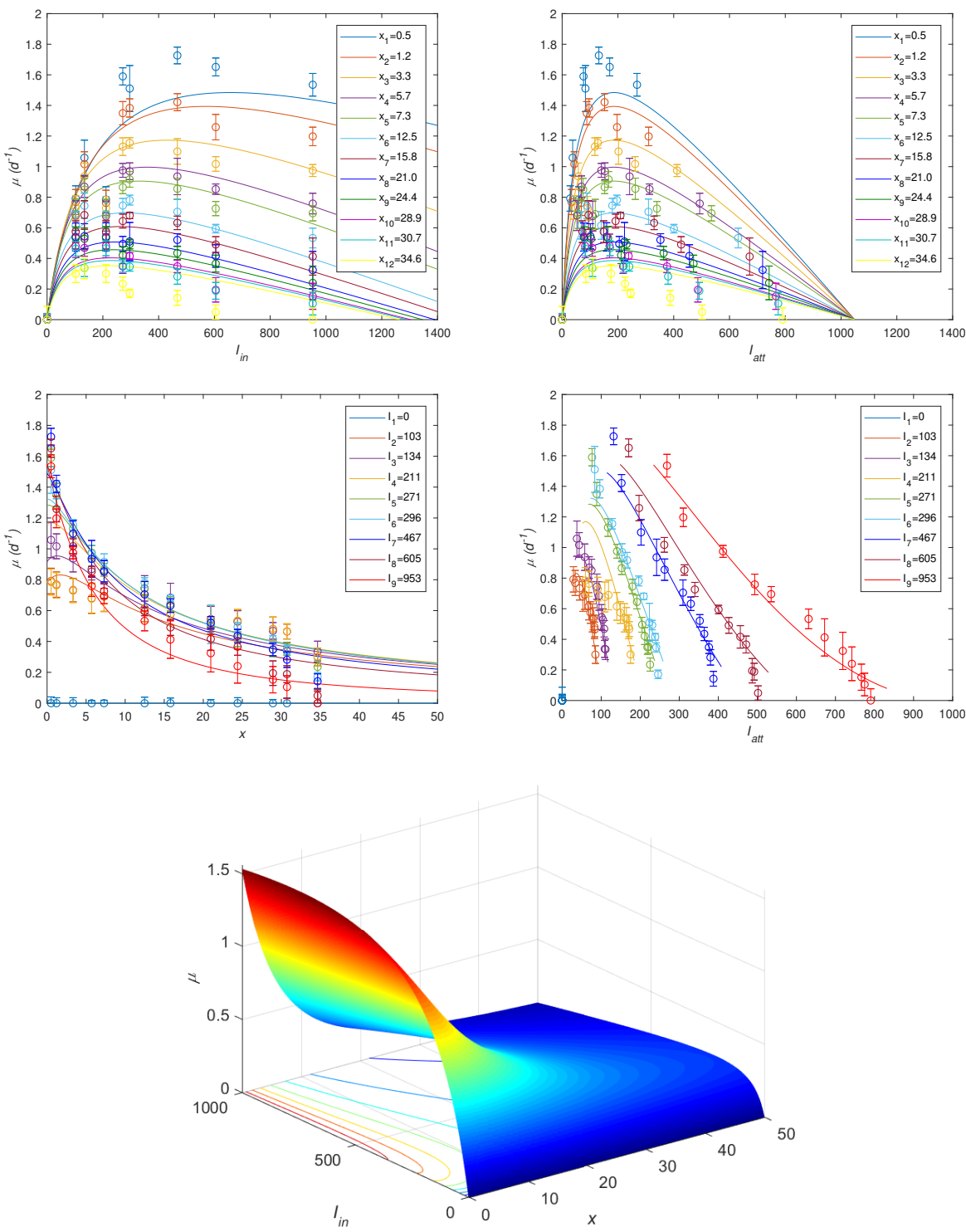

Figure 4: The effect of incident light intensities $I_{i n}\left(\mu \mathrm{Em}^{-2} s^{-1}\right)$ and the biomass densities $x\left(\mathrm{mgC} \mathrm{L}^{-1}\right)$ on the growth of $C$. sorokiniana in microwell plates. The data of growth rates are reported as means with standard deviations of 8 replicate measurements.

dent light and the population density. It reflects the effect of the availability of light, and describes different phenomena that may occur during algal cul- 

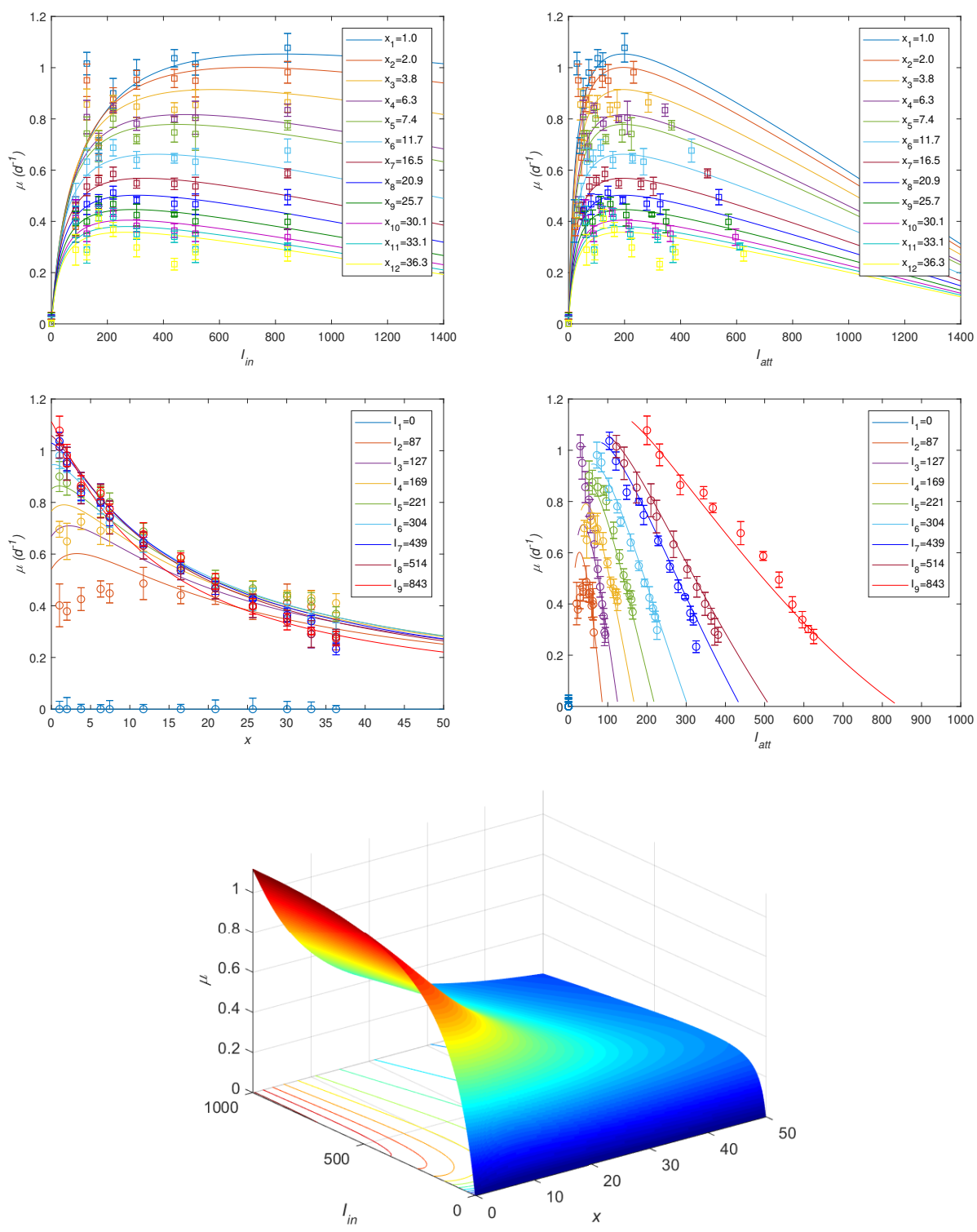

Figure 5: The effect of incident light intensities $I_{i n}\left(\mu E m^{-2} s^{-1}\right)$ and the biomass densities $x\left(\mathrm{mgC} \mathrm{L}^{-1}\right)$ on the growth of $S$. pectinatus in microwell plates. The data of growth rates are reported as means with standard deviations of 8 replicate measurements.

tivation, such as photo-inhibition (following exposure to high light intensities at low biomass concentrations) and photo-limitation (under insufficient 
light conditions), or, more likely, other mechanisms such as cell distribution within water volume. This model requires a limited number of strain-specific parameters and allows comparisons of species growth performances and optimization of the operational parameters of algal cultures. Its simplicity makes it a valuable tool that can be integrated into any type of photo-bioreactor geometry and can apply to a microwell plate (as shown here) or to Erlenmeyer photo-bioreactor (as shown below). Such a growth function also offers a tool for simulating and predicting the potential production rate in poly-culture of different species in algal mass culture systems under light fluctuations (as further explored).

\subsection{Model calibration and extension for poly-culture predictions in contin-} uous mode photo-bioreactors

We considered the data of species growing in mono-culture (in an Erlenmeyer photo-bioreactor exposed to continual $I_{\text {in }}$ ) to compare them to the data generated by the growth kinetics derived by the proposed growth function (6) for growth limited by light. We first need the usual mass balanced model to describe the time evolution of the biomass concentration [49] using the proposed kinetic function $\mu(\cdot)$ from (6) for a fixed intensity of incident light $I_{\text {in }}$.

$$
\dot{x}=\left(\mu\left(I_{i n}, x\right)-D\right) x
$$

In any case, when $D$ is such $0<D<\mu\left(I_{i n}, 0\right)$, the system admits an unique positive equilibrium $x^{\star}$ which belongs to $(0, \bar{x})$, and that is globally symptomatically stable (see Appendix).

The simulations of this model for each species grown in mono-culture are presented in Figure 6 against the data of biomass (from $\mathrm{OD}_{650}$ measurements being converted to $\mathrm{mgC} \mathrm{L}^{-1}$ ) obtained under continuous mode cultures, using the same kinetic parameters represented in Table 3 except for $\alpha$ and $K_{h s x}$. These two parameters are likely to vary considerably depending on the operating conditions. Then, they were both re-identified for model calibration, depending on the culture device being used. $\alpha$ which depends on the culture device, was found to be equal to 0.44 , while $K_{h s x}$, apparently sensitive with regard to mixing, was equal to 14 and $52 \mathrm{mgC} \mathrm{L}^{-1}$ for C. sorokiniana and $S$. pectinatus, respectively. The parameters $\bar{\mu}, K_{s I_{a t t}}$ and $I_{0}$, considered as characteristic parameters of the species, were held the same (as given in Table 3).

In the second step, we sought to validate our growth function (6) on another data set. So, we used the experimental data of biomass (from cell count 

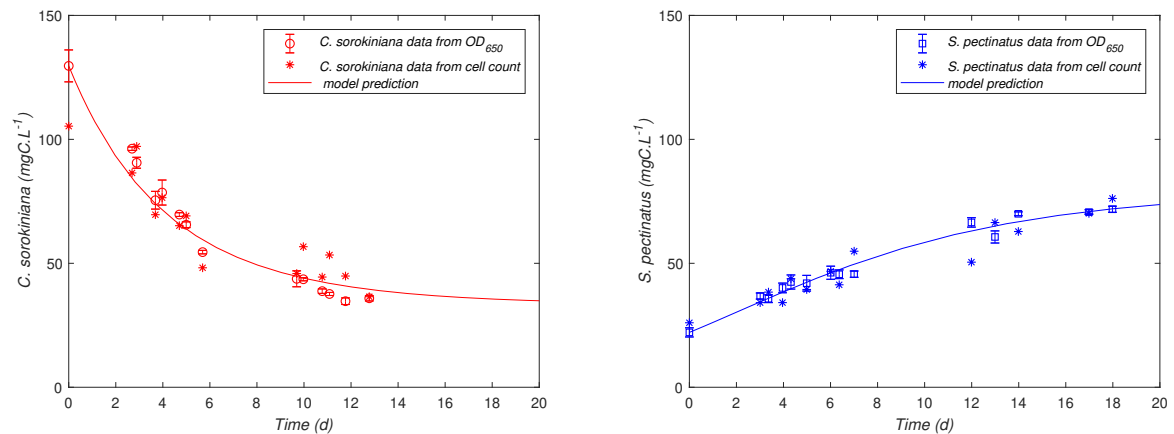

Figure 6: Simulation of chemostat model using the new kinetic function compared to biomass data (means with standard deviations of 3 replicate measurements of $\mathrm{OD}_{650}$ and cell count converted to $\mathrm{mgC} \mathrm{L}^{-1}$ ) tracked in mono-cultures of $C$. sorokiniana and $S$. pectinatus under similar conditions of incident light intensity $I_{\text {in }}=165 \pm 5 \mu \mathrm{E} \mathrm{m}^{-2} \mathrm{~S}^{-1}$ and dilution rate $D=0.25 \pm 0.02 \mathrm{~d}^{-1}$ (in Erlenmeyer photo-bioreactors).

measurements being converted to $\mathrm{mgC}^{-1}$ ) tracked over time in the same Erlenmeyer photo-bioreactor but inoculated with a culture of a mixture of the species. This required an extension of the model to multi-species growths. In this case, the model should be applied in such a way that it would consider species with very different properties, particularly the pigment absorbance, which would be given by the following system

$$
\left\{\begin{array}{l}
\dot{x}_{1}=\left(\mu_{1}\left(I_{i n}, x_{1}+\gamma_{2 / 1} x_{2}\right)-D\right) x_{1} \\
\dot{x}_{2}=\left(\mu_{2}\left(I_{i n}, \gamma_{1 / 2} x_{1}+x_{2}\right)-D\right) x_{2}
\end{array}\right.
$$

where $\gamma_{j / i}$ is the ratio of transparency of species $j$ related to species $i$ with $\left.\gamma_{j / i} \gamma_{i / j}\right)$.

Differently, under the same circumstances, changing the wavelength used for the biomass estimates through correlation with OD could be even more convenient, leading to the simplified system 9 .

$$
\left\{\begin{array}{l}
\dot{x}_{1}=\left(\mu_{1}\left(I_{i n}, x_{1}+x_{2}\right)-D\right) x_{1} \\
\dot{x}_{2}=\left(\mu_{2}\left(I_{i n}, x_{1}+x_{2}\right)-D\right) x_{2}
\end{array}\right.
$$

We suppose that (9) can be applied based on the obtained 'average' correlation between optical density $\left(\mathrm{OD}_{650}\right)$ and the cells carbon content of the studied species in both monoculture and polyculture (cf. Section 2.3), suggesting an equivalent total pigment content for the same biomass concen- 
tration among species belonging to the same family and having experienced similar environmental conditions (leading to consider $\gamma_{2 / 1}=\gamma_{1 / 2}=1$ ).

The same parameters (applied in mono-culture) were used to simulate (9), considering both species growing together (let us underline that these kinetics are coupled here, but differently than the usual interaction terms, such as in the generalized Lotka-Volterra models), and taking into account the nonlinear functions $\mu_{i}$.

The superimposition of the data on the predictions of model (9) in Figure 7 allows a satisfactory description of the dynamics of the different concentrations of the two species. However, it takes a long time to see S. pectinatus stabilizing and C. sorokiniana washed out.

The experiments in continuous photo-bioreactors seem to be explained by the proposed kinetic function when applied in monoculture and polyculture, but new experiments would be necessary for a further validation of the model.

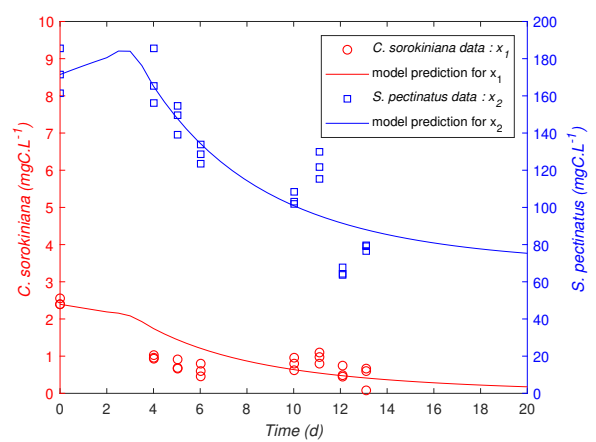

Figure 7: Validation of the chemostat model using the new kinetic function on biomass data (3 replicate measurements of cell count converted to $\mathrm{mgC} \mathrm{L}^{-1}$ ) tracked in species assemblage of $C$. sorokiniana and $S$. pectinatus under a fixed incident light intensity $I_{\text {in }}=$ $165 \pm 5 \mu \mathrm{E} \mathrm{m}^{-2} \mathrm{~s}^{-1}$ and a piece-wise constant dilution rate $D=0.11 \mathrm{~d}^{-1}$ for $t<2.74$; then $D=0.25 \pm 0.02 \mathrm{~d}^{-1}$ (in Erlenmeyer photo-bioreactors).

3.6. Prediction of the possible outcomes of the competition for light availability in continuous mode photo-bioreactors under periodic light conditions

While competitive exclusion is more likely to occur at the laboratory scale [50], the coexistence of species is observed in both natural and artificial ecosystems and may play an important role in the resilience of cultivation systems or even in reducing the risk of extinction under particular conditions 
$[51,52]$. In this section, we discuss three possible outcomes of the multispecies model, including the possibility of species coexistence, through theoretical prediction under periodic light, as a more realistic growth condition. The different situations were corroborated by some simulations (presented in Figure 8) obtained using the growth characteristics previously validated for C. sorokiniana (species 1) and S. pectinatus (species 2) in an Erlenmeyer photo-bioreactor (see Section 3.5), but under different operating conditions (as stated in Table 4).

We recall that the specific growth rate of each species in the multi-species model (9) is influenced by the total biomass density of both species contributing together to attenuate the available light within the photo-bioreactor. Thus, the expressions for $\mu_{1}$ and $\mu_{2}$ in the assemblage depend on the total biomass $x_{1}+x_{2}$ instead of $x_{i}$ only, leading to the model (9) that couples the growth of each species. However, for constant incident light $I_{i n}$, one can easily see that coexistence at steady state is generically impossible, because it would need to have very particular values of $D$ such that the graphs of $\mu_{1}$ and $\mu_{2}$ intersect with a common value exactly equal to $D$. Indeed, this model satisfies the Competitive Exclusion Principle in a very similar way to the classical multi-species chemostat model, for which the common resource is a limiting substrate [49] (to be replaced here by the total biomass). Considering the biomass at steady state in monoculture, denoted by $x_{i}^{\star}$, which satisfies the equation $\mu_{i}\left(x_{i}^{\star}\right)=D$ (recall from the Appendix that in any case, for any $D$ such that $0<D<\mu\left(I_{i n}, 0\right)$, the dynamics $(7)$ admits an unique positive equilibrium, that is globally attractive and stable on the positive domain), the winner of the competition is the species with the largest $x_{i}^{\star}$. This competitive exclusion was observed experimentally under constant light in Section 3.5 (see Figure 7). We note that S. pectinatus won the competition, reaching a value at steady state $x_{2}^{*}$ which verifies $\mu\left(x_{2}^{\star}\right)=D$, as predicted by the competitive exclusion principle.

Let us now consider a periodic $I_{i n}($.$) as a time-varying function. The$ competitive exclusion principle no longer applies. When the input nutrient fluctuates with time (with variable input concentration or variable input flow rate), it is known that species coexistence is possible $[53,54,55]$. Let us see that a similar phenomenon can occur when the incident light is fluctuating (even though the dependency in $I_{i n}$ is non-linear, unlike $D$ ).

We consider first mono-cultures under periodic light:

$$
\dot{x}_{i}=\left(\mu_{i}\left(I_{\text {in }}(t), x_{i}\right)-D\right) x_{i}, \quad i=1,2
$$


It is easy to see that when the condition

$$
C_{i}:=\int_{t}^{t+T}\left(\mu_{i}\left(I_{i n}(\tau), 0\right)-D\right) d \tau>0
$$

is fulfilled, the washout solution $x_{i}=0$ is repelling, and that the scalar dynamics (10) admits an unique positive periodic solution $\tilde{x}_{i}(\cdot)$ (see, for example, the simulations in Figures 8(A), 8(C), 8(E) and 8G, corresponding to mono-cultures obtained under different operating conditions given in Table $4)$, which is asymptotically attractive for any initial condition with $x_{i}(0)>0$ (as $\mu_{i}$ is decreasing with respect to $x_{i}$ ).

Now, consider the co-culture under periodic light:

$$
\left\{\begin{array}{l}
\dot{x}_{1}=\left(\mu_{1}\left(I_{i n}(t), x_{1}+x_{2}\right)-D\right) x_{1} \\
\dot{x}_{2}=\left(\mu_{2}\left(I_{i n}(t), x_{1}+x_{2}\right)-D\right) x_{2}
\end{array}\right.
$$

the asymptotic solutions with the absence of one species, which are $\left(\tilde{x}_{1}(\cdot), 0\right)$ and $\left(0, \tilde{x}_{2}(\cdot)\right)$, are both repelling for the dynamics $(11)$ when conditions

$$
\begin{aligned}
& C_{21}:=\int_{t}^{t+T}\left(\mu_{2}\left(I_{i n}(\tau), \tilde{x}_{1}(\tau)\right)-D\right) d \tau>0 \\
& C_{12}:=\int_{t}^{t+T}\left(\mu_{1}\left(I_{i n}(\tau), \tilde{x}_{2}(\tau)\right)-D\right) d \tau>0
\end{aligned}
$$

are both fulfilled. This argumentation that demonstrates the coexistence of species is often used in the mathematical theory of the chemostat, see for instance $[54,55]$. Let us give some insight into these quantities. When a single species $i$ settles, its concentrations converge with time towards an unique periodic solution $\tilde{x}_{i}(\cdot)$ as previously recalled. When this periodic solution is reached (or almost reached), consider at time $t$ an invasion by the other species $j \neq i$ with a small concentration $x_{j}(t)$. From equations (11), one can see that the time derivative $\dot{x}_{j}$ is small when $x_{j}$ is small. Therefore, if the invasion is such that $x_{j}(t)$ is sufficiently small, $x_{j}$ remains small during the time period $T$, and consequently, the concentration $x_{i}$ is very little impacted while $x_{j}$ remains small. Then, one can assume that $x_{i}(\cdot)$ remains close to the periodic solution $\tilde{x}_{i}(\cdot)$ on the time interval $[t, t+T]$, and the dynamics of $x_{j}$ can be approximated by

$$
\dot{x}_{j}(\tau)=\left(\mu_{j}\left(I_{i n}(\tau), \tilde{x}_{i}(\tau)\right)-D\right) x_{j}(\tau), \quad \tau \in[t, t+T]
$$


whose solution is given by the expression

$$
x_{j}(t+T)=x_{j}(t) \exp \left(\int_{t}^{t+T}\left(\mu_{j}\left(I_{i n}(\tau), \tilde{x}_{i}(\tau)\right)-D\right) d \tau\right)=x_{j}(t) \exp \left(C_{j i}\right)
$$

If $C_{j i}<0$, one has thus $x_{j}(t+T)<x_{j}(t)$ and one can iterate this calculation on the next time interval $[t+T, t+2 T]$ and so on. We conclude that the species $j$ cannot grow. In contrast, when $C_{j i}>0$, species $j$ grows, and its concentration cannot remains close to 0 . We conclude that species $j$ settles in the ecosystem. If the symmetric condition $C_{i j}>0$ is fulfilled for species $i$, we conclude that neither concentration $x_{i}, x_{j}$ can approach 0 . Then, there is necessarily the coexistence of species. This case was illustrated by the example $E_{1}$ in Table 4 and the corresponding simulation presented in Figure 8 (B). By testing multiple values of operating conditions, simulation results show that coexistence is possible, although not systematic. The values of $C_{21}$ and $C_{12}$ can be interpreted as the 'specific invasion speed over one period' and their sign reflects the ability of one species to invade the ecosystem (with small initial density) when the other species is already settled in the periodic regime. Moreover, the magnitudes of $C_{12}$ and $C_{21}$ provide information about the reactivity of the ecosystem to an invasion: the more positive $C_{j i}$ is, the faster is the invasion by the species $j$, and conversely the more negative $C_{j i}$ is, the faster species $j$ is eradicated by the system.

Let us underline the necessity to have the growth functions $\mu_{i}$ alternating its dominance depending on the light to have these two conditions verified. If not, one has for instance $\mu_{1}\left(I_{i n}(t)-x\right)>\mu_{2}\left(I_{i n}(t)-x\right)$ for any $t$ and any $x>0$, which implies

$$
\int_{t}^{t+T}\left(\mu_{1}\left(I_{i n}(\tau), \tilde{x}_{1}(\tau)\right)-D\right) d \tau=0>\int_{t}^{t+T}\left(\mu_{2}\left(I_{\text {in }}(\tau), \tilde{x}_{1}(\tau)\right)=C_{21}\right.
$$

and then $C_{21}>0$ cannot be fulfilled and species 2 cannot invade the system when species 1 is present (see example $E_{2}$ in Table 4 and the corresponding simulation in Figure 8 (D)). Conversely, species 1 cannot invade a culture with species 2 when $C_{12}<0$ (see examples $E_{3}$ and $E_{4}$ in Table 4 and the corresponding simulations for the assemblages in Figures $8(\mathrm{~F})$ and $8(\mathrm{H})$ ). These results show that the coexistence or exclusion of one species or the other are possible depending on the operating conditions $I_{i n}(\cdot)$ and $D$.

We note that the chosen values of the parameters in examples $E_{1}, E_{2}$ and $E_{3}$ in Table 4 are easy to implement at the laboratory scale for operating indoor photo-biorectors. We propose that the model can also apply to 


\begin{tabular}{|c|c|c|c|c|c|c|c|c|c|c|}
\hline \multirow[t]{2}{*}{ Ex. } & \multicolumn{4}{|c|}{ Parameter } & \multicolumn{4}{|c|}{ Test condition } & \multirow[t]{2}{*}{ Outcome } & \multirow[t]{2}{*}{ Fig. } \\
\hline & $\begin{array}{l}T \\
(d)\end{array}$ & $\begin{array}{c}D \\
\left(\mathrm{~d}^{-1}\right)\end{array}$ & $\begin{array}{l}I_{\min } \\
(\mu \mathrm{E} \mathrm{m}\end{array}$ & $\begin{array}{c}I_{\max } \\
\left.-2 \mathrm{~S}^{-1}\right)\end{array}$ & $C_{1}$ & $C_{2}$ & $C_{21}$ & $C_{12}$ & & \\
\hline E1 & 8 & 0.427 & 340 & 1510 & 0.68 & 0.16 & 0.001 & 0.02 & coexist. & $8(\mathrm{~A}, \mathrm{~B})$ \\
\hline E2 & 12 & 0.45 & 52 & 280 & 2.43 & 0.13 & -0.62 & 1.97 & 1 wins & $8(\mathrm{C}, \mathrm{D})$ \\
\hline E3 & 10 & 0.25 & 400 & 900 & 4.57 & 2.60 & 0.89 & -1.05 & 2 wins & $8(\mathrm{E}, \mathrm{F})$ \\
\hline E4 & 1 & 0.16 & 0 & 700 & 0.19 & 0.09 & 0.03 & -0.05 & 2 wins & $8(\mathrm{G}, \mathrm{H})$ \\
\hline
\end{tabular}

Table 4: Some illustrative examples of the possible outcomes of the multispecies model using different operational conditions of dilution rate $D$ and periodic illumination (taking $I_{\min }$ and $I_{\max }$ over the period $T$ ). The test conditions $C_{1}$ and $C_{2}$ are computed on species 1 ( C. sorokiniana) and species 2 (S. pectinatus) in monoculture, while $C_{12}$ and $C_{21}$ are given for assemblages. The other parameters are taken to be: $\alpha=0.4$; For species 1 : $\bar{\mu}=3.25 \mathrm{~d}^{-1}, K_{s I_{a t t}}=52 \mu \mathrm{E} \mathrm{m}^{-2} \mathrm{~s}^{-1}, K_{h s x}=52 \mathrm{mgC} \mathrm{L}^{-1}$, and $I_{0}=1068 \mu \mathrm{E} \mathrm{m}^{-2} \mathrm{~s}^{-1}$; For species $2: \bar{\mu}=1.75 \mathrm{~d}^{-1}, K_{s I_{a t t}}=26 \mu \mathrm{E} \mathrm{m}^{-2} \mathrm{~s}^{-1}, K_{h s x}=26 \mathrm{mgC} \mathrm{L}^{-1}$, and $I_{0}=1836$ $\mu \mathrm{E} \mathrm{m}^{-2} \mathrm{~s}^{-1}$.

outdoor cultures. For such a case, we considered in $E_{4}$ (in Table 4 ) more appropriate conditions for the light for simulating the light-dark cycles, which may be given with an illumination fluctuating between $I_{\min }=0$ and an average value $I_{\max }$ (at about $700 \mu \mathrm{E} \mathrm{m}^{-2} \mathrm{~s}^{-1}[36,56]$ ) over a period $T$ of one day. Under these latter conditions, the model (11) theoretically predicted a competitive exclusion in favor of $S$. pectinatus, as shown in Figure $8(\mathrm{H})$. The predominance of Scenedesmus predicted by the simulation reproduces the experimental observations of several studies [57, 58, 36, 37, 56].

One can notice in Figure 8 that during the transients, the densities of both species increase (or decrease) simultaneously before one of them reaches a stage from which it declines. This is qualitatively different from the transients of the exclusion obtained with the classical model of competition on an abiotic resource (such as limited substrate) described by the usual growth functions $[55,49,37]$. This feature could be a matter for future research to discriminate which kind of exclusion (due to light or substrate) is dominant, and when.

\section{Conclusion}

Light inhibition and attenuation appear to have significant effects on the growth of micro-algae. The presented results show that the reduction of species growth rates was mainly attributed to high cell densities, which reduce the penetration of light into the culture, but may protect cells from 

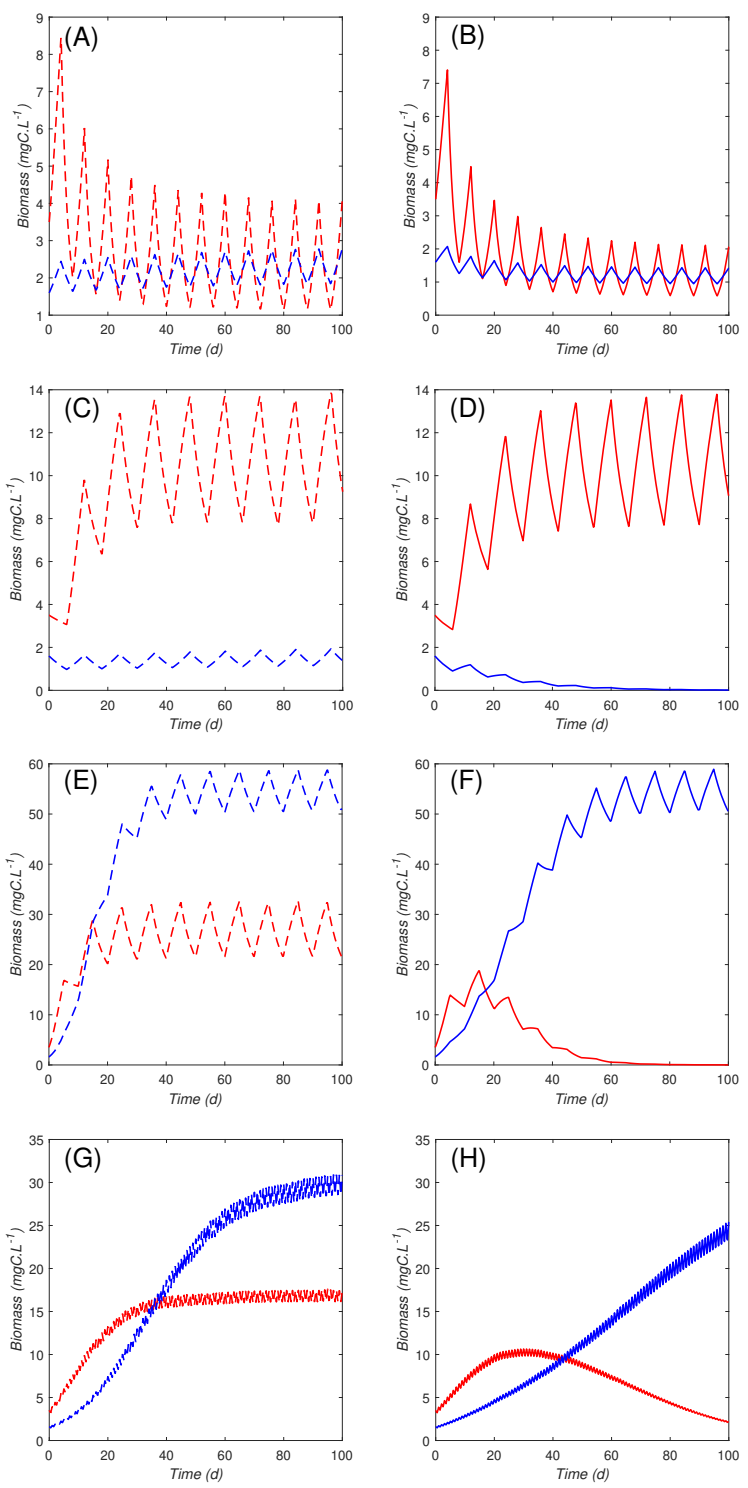

Figure 8: Some illustrative simulations obtained in continuous mode photo-bioreactors exposed to periodic illumination in mono-culture (first column A, C, E and G) and in assemblage (second column B, D, F and H) for species 1 (C. sorokiniana) (in red) and species 2 (S. pectinatus) (in blue) according to the examples of operational conditions stated in Table 4. 
photo-inhibition when exposed to high light levels. S. pectinatus demonstrated better performances than $C$. sorokiniana at insufficient or excessive light availability, while $C$. sorokiniana was able to achieve faster growth under non-inhibiting light levels in clearer waters. We have shown that the newly developed kinetic model, depending on both the incident light and the biomass densities through the attenuated light model, can describe the simultaneous effects of photo-inhibition and photo-limitation and predict the biomass production in mono-culture and species assemblage. The use of modeling and experimental approaches allows the characterization of the species and the proper model identification for estimating the biomass production under different operating conditions and assessing the optimal operational parameters, which is of great benefit for the evaluation of a small or large scale algal mass culture, particularly in poly-culture systems.

This new model offers various possible future applications, such as its use for automatic monitoring of the instantaneous biomass concentration through light measurements within the reactor, or even the effective optimization of the incident light intensities, in addition to possible control (playing with the light availability in indoor cultures or shadowing in outdoor culture). The control of the incident light, the dilution rate, and the choice of initial biomass for the optimization of productivity in poly-culture will need further investigation.

\section{Appendix: study of the behavior of the function $\mu$}

We study the monotonicity of the function $\mu$ with respect to $x$, for a fixed given value of $I_{i n}>0$. For this purpose, let us posit

$$
X:=I_{a t t}\left(I_{i n}, x\right)=I_{\text {in }}\left(1-\alpha\left(1-\frac{x}{x+K_{h s x}}\right)\right)
$$

(where $\alpha \in(0,1)$ and $K_{h s x}>0$ ). One can straightforwardly see that $X$ is an increasing function of $x \geq 0$, that takes values in $\left[I_{i n}(1-\alpha), I_{i n}\right]$. Then, the function $\mu$ can be expressed as a function of $X$ as follows

$$
\mu\left(I_{i n}, x\right)=\tilde{\mu}(X):=A(X) B(X)
$$

with

$$
A(X)=\bar{\mu}\left(1-\frac{X}{I_{\text {in }}}\right)\left(1-\frac{X}{I_{0}}\right), \quad B(X)=\frac{X}{K_{\text {sIatt }}+X}
$$


(where $I_{0}>0$ and $K_{\text {sIatt }}>0$ ). Remind that one has $(1-\alpha)<I_{0}<I_{\text {in }}$ in the model. The validity domain of the expression (14) is for $X$ such that $\mu(X) \geq 0$, that is for $X \in \mathcal{D}:=\left[I_{i n}(1-\alpha), I_{0}\right]$, or equivalently for $x$ such that

$$
x \leq \bar{x}:=K_{h s x} \frac{I_{0}-(1-\alpha) I_{i n}}{I_{\text {in }}-I_{0}}
$$

(for value of $x$ above $\bar{x}$, we consider that $\mu$ is equal to 0 ). Note that the function $A$ is convex quadratic and decreasing non-negative on $\mathcal{D}$, and that $B$ is a concave increasing function. Furthermore, one can easily check that the function $B$ satisfies $B^{\prime \prime \prime}>0$. By Leibniz rule, one get

$$
\tilde{\mu}^{\prime \prime \prime}(X)=3 A^{\prime \prime}(X) B^{\prime}(X)+3 A^{\prime}(X) B^{\prime \prime}(X)+A(X) B^{\prime \prime}(X)>0, \quad X \in \mathcal{D}
$$

Therefore, $\tilde{\mu}$ can change its concavity on $\mathcal{D}$ only from concave to convex. If $\tilde{\mu}$ is convex on $\mathcal{D}$, it has to be decreasing on $\mathcal{D}$ because $I_{0}$ is the first zero. Otherwise, it is concave at $I_{i n}(1-\alpha)$. Then, it is decreasing on $\mathcal{D}$ or there exists $\bar{X} \in\left(I_{\text {in }}(1-\alpha), I_{0}\right)$ such that $\tilde{\mu}$ is increasing on $\left(I_{\text {in }}(1-\alpha), \bar{X}\right)$ and decreasing on $\left(\bar{X}, I_{0}\right)$.

In any case, for any $D$ such that $0<D<\tilde{\mu}\left(I_{\text {in }}(1-\alpha)\right)=\mu\left(I_{i n}, 0\right)$, the dynamics

$$
\dot{x}=\left(\mu\left(I_{i n}, x\right)-D\right) x
$$

admits an unique positive equilibrium, that is globally attractive and stable on the positive domain.

\section{Aknowledgements}

We thank Patrick Raimbault for carbon content analyses, Tatiana Linhart for cell count, and Axelle Menard for giving a helping hand to experiments monitoring. We would also like to thank Bénédicte Fontez for assistance with statistical analyses.

This work was supported by the ADEME French Agency and the LabEx NUMEV incorporated into the I-Site MUSE funded by the French Research Agency (ANR) that have both funded the Ph.D. grant of the first author. This study was also supported by the PHYCOVER project, which was funded by the French National Agency for Research (ANR-14-CE04-0011).

\section{Statement of informed consent, human/animal rights}

No conflicts, informed consent, human or animal rights applicable. 


\section{References}

[1] S. R. Medipally, F. M. Yusoff, S. Banerjee, M. Shariff, Microalgae as sustainable renewable energy feedstock for biofuel production, BioMed research international 2015 (2015).

[2] V. Makareviciene, V. Andrulevičiūtè, V. Skorupskaitè, J. Kasperovičienè, Cultivation of microalgae chlorella sp. and scenedesmus sp. as a potentional biofuel feedstock, Environmental Research, Engineering and Management 57 (2011) 21-27.

[3] J. Koreivienè, R. Valčiukas, J. Karosienè, P. Baltrènas, Testing of chlorella/scenedesmus microalgae consortia for remediation of wastewater, co2 mitigation and algae biomass feasibility for lipid production, Journal of Environmental Engineering and Landscape Management 22 (2014) 105-114.

[4] J. Jena, M. Nayak, H. S. Panda, N. Pradhan, C. Sarika, P. K. Panda, B. Rao, R. B. Prasad, L. B. Sukla, Microalgae of odisha coast as a potential source for biodiesel production, World Environ 2 (2012) 1116.

[5] S. B. Ummalyma, D. Sahoo, A. Pandey, Bioremediation and biofuel production from chlorella sp.: A comprehensive review, in: Microalgae Biotechnology for Development of Biofuel and Wastewater Treatment, Springer, 2019, pp. 635-655.

[6] A. Parmar, N. K. Singh, A. Pandey, E. Gnansounou, D. Madamwar, Cyanobacteria and microalgae: a positive prospect for biofuels, Bioresource technology 102 (2011) 10163-10172.

[7] A. P. Carvalho, S. O. Silva, J. M. Baptista, F. X. Malcata, Light requirements in microalgal photobioreactors: an overview of biophotonic aspects, Applied microbiology and biotechnology 89 (2011) 1275-1288.

[8] M. Al-Qasmi, N. Raut, S. Talebi, S. Al-Rajhi, T. Al-Barwani, A review of effect of light on microalgae growth, in: Proceedings of the world congress on engineering, volume 1, 2012, pp. 4-6.

[9] I. Krzemińska, B. Pawlik-Skowrońska, M. Trzcińska, J. Tys, Influence of photoperiods on the growth rate and biomass productivity of green microalgae, Bioprocess and biosystems engineering 37 (2014) 735-741. 
[10] S. P. Long, S. Humphries, P. G. Falkowski, Photoinhibition of photosynthesis in nature, Annual review of plant biology 45 (1994) 633-662.

[11] N. K. Singh, D. W. Dhar, Microalgae as second generation biofuel. a review, Agronomy for Sustainable Development 31 (2011) 605-629.

[12] H. Jeong, J. Lee, M. Cha, Energy efficient growth control of microalgae using photobiological methods, Renewable energy 54 (2013) 161-165.

[13] R. Geider, H. MacIntyre, T. Kana, Dynamic model of phytoplankton growth and acclimation: responses of the balanced growth rate and the chlorophyll a: carbon ratio to light, nutrient-limitation and temperature, Marine Ecology Progress Series 148 (1997) 187-200.

[14] O. Bernard, Hurdles and challenges for modelling and control of microalgae for co2 mitigation and biofuel production, Journal of Process Control 21 (2011) 1378-1389.

[15] P. G. Falkowski, J. LaRoche, Acclimation to spectral irradiance in algae, Journal of Phycology 27 (1991) 8-14.

[16] T. Anning, H. L. MacIntyre, S. M. Pratt, P. J. Sammes, S. Gibb, R. J. Geider, Photoacclimation in the marine diatom skeletonema costatum, Limnology and Oceanography 45 (2000) 1807-1817.

[17] H. L. MacIntyre, T. M. Kana, T. Anning, R. J. Geider, Photoacclimation of photosynthesis irradiance response curves and photosynthetic pigments in microalgae and cyanobacteria 1, Journal of phycology 38 (2002) 17-38.

[18] F. C. Rubio, F. G. Camacho, J. F. Sevilla, Y. Chisti, E. M. Grima, A mechanistic model of photosynthesis in microalgae, Biotechnology and bioengineering 81 (2003) 459-473.

[19] P. Hartmann, A. Nikolaou, B. Chachuat, O. Bernard, A dynamic model coupling photoacclimation and photoinhibition in microalgae, in: 2013 European Control Conference (ECC), IEEE, 2013, pp. 4178-4183.

[20] A. Nikolaou, P. Hartmann, A. Sciandra, B. Chachuat, O. Bernard, Dynamic coupling of photoacclimation and photoinhibition in a model of microalgae growth, Journal of theoretical biology 390 (2016) 61-72. 
[21] J. Monod, Recherches sur la croissance des cultures bacteriennes, Ph.D. thesis, 1942.

[22] J.-F. Cornet, C. Dussap, J.-B. Gros, C. Binois, C. Lasseur, A simplified monodimensional approach for modeling coupling between radiant light transfer and growth kinetics in photobioreactors, Chemical Engineering Science 50 (1995) 1489-1500.

[23] Y.-C. Jeon, C.-W. Cho, Y.-S. Yun, Measurement of microalgal photosynthetic activity depending on light intensity and quality, Biochemical Engineering Journal 27 (2005) 127-131.

[24] J. F. Andrews, A mathematical model for the continuous culture of microorganisms utilizing inhibitory substrates, Biotechnology and Bioengineering 10 (1968) 707-723.

[25] T. Ogawa, S. Aiba, Bioenergetic analysis of mixotrophic growth in chlorella vulgaris and scenedesmus acutus, Biotechnology and Bioengineering 23 (1981) 1121-1132.

[26] R. Megard, D. Tonkyn, W. Senft, Kinetics of oxygenic photosynthesis in planktonic algae, Journal of Plankton Research 6 (1984) 325-337.

[27] J. Steele, Microbial kinetics and dynamics in chemical reactor theory, in: Chemical reactor theory, Englewood Cliffs, NJ, 1977, pp. 405-483.

[28] E. M. Grima, J. F. Sevilla, J. S. Pérez, F. G. Camacho, A study on simultaneous photolimitation and photoinhibition in dense microalgal cultures taking into account incident and averaged irradiances, Journal of Biotechnology 45 (1996) 59-69.

[29] Q. Béchet, A. Shilton, B. Guieysse, Modeling the effects of light and temperature on algae growth: state of the art and critical assessment for productivity prediction during outdoor cultivation, Biotechnology advances 31 (2013) 1648-1663.

[30] P. Darvehei, P. A. Bahri, N. R. Moheimani, Model development for the growth of microalgae: A review, Renewable and Sustainable Energy Reviews 97 (2018) 233-258. 
[31] S. Aiba, Growth kinetics of photosynthetic microorganisms, in: Microbial reactions, Springer, 1982, pp. 85-156.

[32] J. Huisman, H. C. Matthijs, P. M. Visser, H. Balke, C. A. Sigon, J. Passarge, F. J. Weissing, L. R. Mur, Principles of the light-limited chemostat: theory and ecological applications, Antonie van Leeuwenhoek 81 (2002) 117-133.

[33] W. E. A. Kardinaal, L. Tonk, I. Janse, S. Hol, P. Slot, J. Huisman, P. M. Visser, Competition for light between toxic and nontoxic strains of the harmful cyanobacterium microcystis, Appl. Environ. Microbiol. 73 (2007) 2939-2946.

[34] F. Grognard, A. R. Akhmetzhanov, O. Bernard, Optimal strategies for biomass productivity maximization in a photobioreactor using natural light, Automatica 50 (2014) 359-368.

[35] S. F. Salleh, A. Kamaruddin, M. H. Uzir, A. R. Mohamed, A. H. Shamsuddin, Modeling the light attenuation phenomenon during photoautotrophic growth of a. variabilis atcc 29413 in a batch photobioreactor, Journal of Chemical Technology \& Biotechnology 92 (2017) 358-366.

[36] A. Galès, A. Bonnafous, C. Carré, V. Jauzein, E. Lanouguère, E. Le Floc'h, J. Pinoit, C. Poullain, C. Roques, B. Sialve, et al., Importance of ecological interactions during wastewater treatment using high rate algal ponds under different temperate climates, Algal Research 40 (2019) 101508.

[37] E. Krichen, A. Rapaport, E. Le Floc'h, E. Fouilland, Demonstration of facilitation between microalgae to face environmental stress, Scientific Reports 9 (2019) 16076.

[38] J. Kotai, Instructions for preparation of modified nutrient solution z8 for algae, Norwegian Institute for Water Research, Oslo 11 (1972) 5.

[39] N. R. Draper, H. Smith, Applied regression analysis, volume 326, John Wiley \& Sons, 1998.

[40] P. Westerhoff, Q. Hu, M. Esparza-Soto, W. Vermaas, Growth parameters of microalgae tolerant to high levels of carbon dioxide in batch and 
continuous-flow photobioreactors, Environmental technology 31 (2010) $523-532$.

[41] M. Huesemann, B. Crowe, P. Waller, A. Chavis, S. Hobbs, S. Edmundson, M. Wigmosta, A validated model to predict microalgae growth in outdoor pond cultures subjected to fluctuating light intensities and water temperatures, Algal Research 13 (2016) 195-206.

[42] J. Masojídek, G. Torzillo, M. Koblížek, J. Kopeckỳ, P. Bernardini, A. Sacchi, J. Komenda, Photoadaptation of two members of the chlorophyta (scenedesmus and chlorella) in laboratory and outdoor cultures: changes in chlorophyll fluorescence quenching and the xanthophyll cycle, Planta 209 (1999) 126-135.

[43] H. Qiang, A. Richmond, Optimizing the population density inisochrysis galbana grown outdoors in a glass column photobioreactor, Journal of Applied Phycology 6 (1994) 391-396.

[44] B.-P. Han, M. Virtanen, J. Koponen, M. Straškraba, Effect of photoinhibition on algal photosynthesis: a dynamic model, Journal of Plankton Research 22 (2000) 865-885.

[45] D. Contois, Kinetics of bacterial growth: relationship between population density and specific growth rate of continuous cultures, Microbiology 21 (1959) 40-50.

[46] Y. Zhao, J. Wang, H. Zhang, C. Yan, Y. Zhang, Effects of various led light wavelengths and intensities on microalgae-based simultaneous biogas upgrading and digestate nutrient reduction process, Bioresource technology 136 (2013) 461-468.

[47] A. Khalili, G. D. Najafpour, G. Amini, F. Samkhaniyani, Influence of nutrients and led light intensities on biomass production of microalgae chlorella vulgaris, Biotechnology and bioprocess engineering 20 (2015) $284-290$.

[48] P. G. Falkowski, Light-shade adaptation in marine phytoplankton, in: Primary productivity in the sea, Springer, 1980, pp. 99-119. 
[49] J. Harmand, C. Lobry, A. Rapaport, T. Sari, Le chémostat: Théorie mathématique de la culture continue de micro-organismes, volume 1, ISTE Group, 2017.

[50] S. Hubbell, et al., Single-nutrient microbial competition: qualitative agreement between experimental and theoretically forecast outcomes, Science 207 (1980) 1491-1493.

[51] G. Peterson, C. R. Allen, C. S. Holling, Ecological resilience, biodiversity, and scale, Ecosystems 1 (1998) 6-18.

[52] F. Jeltsch, V. Grimm, J. Reeg, U. E. Schlägel, Give chance a chance: from coexistence to coviability in biodiversity theory, Ecosphere 10 (2019) e02700.

[53] S.-B. Hsu, A competition model for a seasonally fluctuating nutrient, Journal of Mathematical Biology 9 (1980) 115-132.

[54] G. Butler, S. Hsu, P. Waltman, A mathematical model of the chemostat with periodic washout rate, SIAM J. Appl. Math. 45 (1985) 435-449.

[55] H. L. Smith, P. Waltman, The theory of the chemostat: dynamics of microbial competition, volume 13, Cambridge university press, 1995.

[56] S.-W. Jo, J.-M. Do, H. Na, J. W. Hong, I.-S. Kim, H.-S. Yoon, Assessment of biomass potentials of microalgal communities in open pond raceways using mass cultivation, PeerJ 8 (2020) e9418.

[57] S. Huo, C. Shang, Z. Wang, W. Zhou, F. Cui, F. Zhu, Z. Yuan, R. Dong, Outdoor growth characterization of an unknown microalga screened from contaminated chlorella culture, BioMed research international 2017 (2017).

[58] D.-H. Cho, J.-W. Choi, Z. Kang, B.-H. Kim, H.-M. Oh, H.-s. Kim, R. Ramanan, Microalgal diversity fosters stable biomass productivity in open ponds treating wastewater, Scientific Reports 7 (2017) 1-11. 